\title{
Topological symmetry groups of graphs embedded in the 3-sphere
}

\author{
Erica Flapan, Ramin Naimi, James Pommersheim and Harry Tamvakis*
}

\begin{abstract}
The topological symmetry group of a graph embedded in the 3-sphere is the group consisting of those automorphisms of the graph which are induced by some homeomorphism of the ambient space. We prove strong restrictions on the groups that can occur as the topological symmetry group of some embedded graph. In addition, we characterize the orientation preserving topological symmetry groups of embedded 3-connected graphs in the 3-sphere.
\end{abstract}

Mathematics Subject Classification (2000). 05C10, 57M15; 05C25, 57M25, 57N10.

Keywords. Topological symmetry group, automorphism group, embedded graph, spatial graph, 3-connected graph.

\section{Introduction}

In this paper, we begin a systematic study of topological symmetry groups of graphs embedded in the 3 -sphere. The notion of a topological symmetry group was introduced by Simon [Si], who was motivated by the Longuet-Higgins symmetry groups of non-rigid molecules [L]. The significance of symmetry groups in chemistry stems from the fact that the chemical properties of a molecule depend on the symmetries of its molecular graph (where the vertices represent atoms and the edges represent bonds).

The study of graphs as geometric objects necessarily involves an investigation of their symmetries. The symmetries of an abstract graph $\gamma$ are described by the group $\operatorname{Aut}(\gamma)$ of automorphisms of $\gamma$. The automorphism group of a graph has been the subject of much study, with roots in the nineteenth century (see [B3] and [B4] for surveys). In contrast, the group of those symmetries of an embedded graph in $S^{3}$ which are induced by homeomorphisms of the ambient space has received little attention.

By a graph we shall mean a finite, connected graph, such that each edge has two distinct vertices and there is at most one edge with a given pair of vertices. An

\footnotetext{
${ }^{*}$ The fourth author was supported in part by NSF Grant DMS-0296023.
} 
embedded graph $\Gamma$ is a pair $(V, E)$ of sets of vertices $V$ and edges $E$ such that $V$ is a set of points in $S^{3}$, every edge is a smoothly embedded $\operatorname{arc}$ in $S^{3}$ between two vertices, and the interior of each edge contains no vertex and no point of any other edge. When we write $h:\left(S^{3}, \Gamma\right) \rightarrow\left(S^{3}, \Gamma\right)$ or $h(\Gamma)=\Gamma$, we shall mean that $h(V)=V$ and $h(E)=E$. The restriction of $h$ to $V$ induces an automorphism of the abstract graph $\gamma$ underlying $\Gamma$. The topological symmetry group $\operatorname{TSG}(\Gamma)$ is defined to be the subgroup of $\operatorname{Aut}(\gamma)$ consisting of those automorphisms which are induced by some homeomorphism of $\left(S^{3}, \Gamma\right)$. Allowing only orientation preserving homeomorphisms of $S^{3}$ defines the orientation preserving topological symmetry group $\mathrm{TSG}_{+}(\Gamma)$. For any embedded graph $\Gamma$, either $\operatorname{TSG}_{+}(\Gamma)=\operatorname{TSG}(\Gamma)$ or $\operatorname{TSG}_{+}(\Gamma)$ is a normal subgroup of TSG $(\Gamma)$ with index 2. Starting with a particular embedded graph $\Gamma$, we can re-embed it by tying the same invertible chiral knot in every edge of $\Gamma$ to get an embedded graph $\Gamma^{\prime}$ such that $\operatorname{TSG}\left(\Gamma^{\prime}\right)=\mathrm{TSG}_{+}\left(\Gamma^{\prime}\right)=\mathrm{TSG}_{+}(\Gamma)$. Thus every group which is the orientation preserving topological symmetry group of some embedded graph is also the topological symmetry group of some (possibly different) embedded graph.

Frucht [Fr] showed that any finite group is the automorphism group of some connected graph; moreover, restricting to $k$-connected graphs for a fixed $k \geq 2$ does not affect the conclusion [Sa] (a graph is $k$-connected if at least $k$ vertices together with their incident edges must be removed in order to disconnect the graph or reduce it to a single vertex). Since every graph admits an embedding in $S^{3}$, it is natural to ask whether every finite group can be realized as $\operatorname{TSG}(\Gamma)\left(\right.$ or $\operatorname{TSG}_{+}(\Gamma)$ ) for some embedded graph $\Gamma$. Using the terminology of [B3], the question becomes whether the class of embedded graphs and their topological symmetry groups is universal for finite groups. We show that the answer to this question is negative, and we characterize the class of all orientation preserving topological symmetry groups for 3-connected graphs.

In general, $\mathrm{TSG}_{+}(\Gamma)$ will depend on the particular embedding of the graph in $S^{3}$. For example, consider $\theta_{n}$ consisting of two vertices which are joined together by $n$ edges. Since $\theta_{n}$ is not a graph, we add a vertex of valence 2 to each edge to obtain a graph $\gamma_{n}$ (see Figure 1). Starting with a planar embedding of $\gamma_{n}$, we add identical

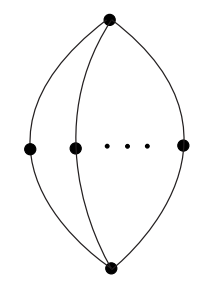

Figure 1. $\gamma_{n}$ 
non-invertible knots to each of the arcs to obtain an embedded graph $\Gamma_{n}$ such that $\mathrm{TSG}_{+}\left(\Gamma_{n}\right)$ is the symmetric group $S_{n}$. On the other hand, if $\Gamma_{n}^{\prime}$ is an embedded graph obtained from a planar embedding of $\gamma_{n}$ by tying distinct non-invertible knots in each edge, then $\mathrm{TSG}_{+}\left(\Gamma_{n}^{\prime}\right)$ is trivial.

Given any finite abelian group $H$, we can construct an embedded graph $\Gamma$ such that $\mathrm{TSG}_{+}(\Gamma)=H$. For example, the embedded graph $\Gamma$ which is illustrated in Figure 2 has $\mathrm{TSG}_{+}(\Gamma)=\mathbb{Z}_{2} \times \mathbb{Z}_{3} \times \mathbb{Z}_{4}$. If $H$ contains more than one factor of a given $\mathbb{Z}_{n}$, we can add knots to the spokes of each "wheel" so that no homeomorphism takes one "wheel" to another "wheel."

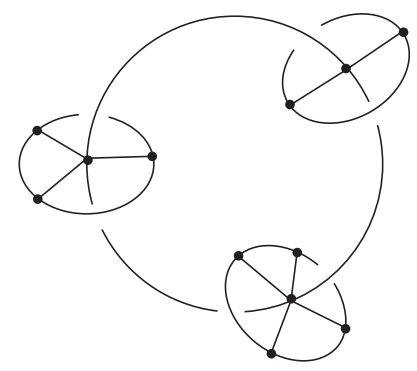

Figure 2. $\mathrm{TSG}_{+}(\Gamma)=\mathbb{Z}_{2} \times \mathbb{Z}_{3} \times \mathbb{Z}_{4}$

Another source of topological symmetry groups comes from planar embeddings. For a planar graph $\gamma$ realized as a planar embedded graph $\Gamma$ via the natural inclusion of $S^{2}$ in $S^{3}$, it can be shown using results of [Ma] and [D, Theorem 4.3.1] that $\mathrm{TSG}(\Gamma)=\mathrm{TSG}_{+}(\Gamma)=\operatorname{Aut}(\gamma)$. The automorphism groups of planar graphs have been characterized by Mani [M] and Babai [B1], [B2]. In particular, these groups do not exhaust all finite groups, and for 3-connected planar graphs the automorphism groups are precisely the finite subgroups of $\mathrm{O}(3)$. In contrast to the case of planar embeddings, in general for an arbitrary embedded graph, $\mathrm{TSG}_{+}(\Gamma) \neq \operatorname{Aut}(\gamma)$. In fact, it was shown in [F1] that for $n \geqslant 6$, no matter how the complete graph $K_{n}$ is embedded in $S^{3}$, the cycle automorphism (1234) of $K_{n}$ cannot be induced by a homeomorphism of $S^{3}$. Thus for any embedded graph $\Gamma$ which has underlying abstract graph $K_{n}$ with $n \geqslant 6, \operatorname{TSG}(\Gamma)$ is a proper subgroup of $\operatorname{Aut}\left(K_{n}\right)$.

In general, it is not possible for each element of $\mathrm{TSG}_{+}(\Gamma)$ to be induced by a finite order homeomorphism of $S^{3}$. For example, consider the graph $\Gamma_{n}$ with $n \geqslant 4$ described above whose underlying abstract graph is illustrated in Figure 1. Then as seen above, $\mathrm{TSG}_{+}\left(\Gamma_{n}\right)=S_{n}$; however, many of the homeomorphisms of $S^{3}$ which induce the elements of $\mathrm{TSG}_{+}(\Gamma)$ cannot be of finite order. Indeed, it follows from the proof of the Smith Conjecture [MB] that no finite order homeomorphism of $\left(S^{3}, \Gamma_{n}\right)$ can interchange two vertices of valence two and fix the remaining vertices, since the fixed point set of such a homeomorphism would include a non-trivial knot. 
In fact, there exist 3-connected embedded graphs $\Gamma$ such that not every element of TSG $(\Gamma)$ can be induced by a finite order homeomorphism of $S^{3}$. An example of such a graph is illustrated in Figure 3. There is no order 3 homeomorphism of $S^{3}$ which takes a figure eight knot to itself ([Ha] and [Tr]); and by Smith Theory [Sm], no finite order homeomorphism of $S^{3}$ can pointwise fix $\Gamma$. Hence the automorphism (123) (456) is induced by a homeomorphism of $S^{3}$ (by sliding the graph along itself), but cannot be induced by a finite order homeomorphism of $S^{3}$.

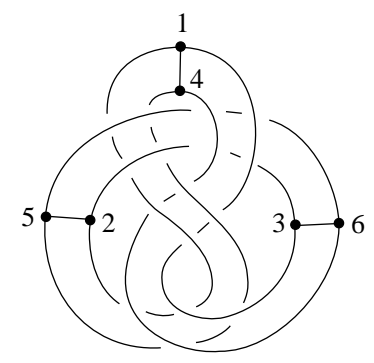

Figure 3. (123)(456) cannot be induced by a finite order homeomorphism of $S^{3}$

The above examples indicate that a priori, the classification of all possible topological symmetry groups could be rather complicated. The three main theorems which follow help to clarify the situation.

Theorem 1. Let $\Gamma$ be an embedded graph.

a) If $\mathrm{TSG}_{+}(\Gamma)$ is a simple group, then it is either the alternating group $A_{5}$ or a cyclic group of prime order.

b) In general, the sequence of quotient groups in any composition series for $\mathrm{TSG}_{+}(\Gamma)$ contains only alternating groups $A_{n}$ with $n \geqslant 5$ and cyclic groups.

We note that the same conclusion holds for the automorphism groups of planar graphs; in fact Theorem 1 implies the corresponding results in [B2], since $\mathrm{TSG}_{+}(\Gamma)=$ $\operatorname{Aut}(\gamma)$ if $\Gamma$ is planar embedding of $\gamma$. However, there exist embedded graphs $\Gamma$ such that $\mathrm{TSG}_{+}(\Gamma)$ is not isomorphic to the automorphism group of any planar graph (see Section 2).

It follows from Theorem 1 that the class of orientation preserving topological symmetry groups of embedded graphs is not universal for finite groups. Furthermore, if $\operatorname{TSG}(\Gamma)$ is a simple group then $\operatorname{TSG}_{+}(\Gamma)=\operatorname{TSG}(\Gamma)$, and hence the class of topological symmetry groups is also not universal.

Theorem 2. Let $\Gamma$ be an embedded 3-connected graph. Then $\mathrm{TSG}_{+}(\Gamma)$ is isomorphic to a finite subgroup of the group Diff $+\left(S^{3}\right)$ of orientation preserving diffeomorphisms of $S^{3}$. 
In contrast to Theorem 2, the orientation preserving topological symmetry group of an arbitrary graph is not necessarily isomorphic to a subgroup of $\operatorname{Diff}_{+}\left(S^{3}\right)$. For example, we have seen that every finite abelian group is the orientation preserving topological symmetry group of some embedded graph. However, not all finite abelian groups are subgroups of $\operatorname{Diff}_{+}\left(S^{3}\right)$.

In the last section of the paper, we study when a graph $\gamma$ may be embedded in $S^{3}$ in such a way that a given subgroup of $\operatorname{Aut}(\gamma)$ is induced on the embedded graph by an isomorphic subgroup of $\operatorname{Diff}_{+}\left(S^{3}\right)$. We also prove the following converse to Theorem 2.

Theorem 3. For every finite subgroup $G$ of $\operatorname{Diff}_{+}\left(S^{3}\right)$, there is an embedded 3connected graph $\Gamma$ such that $G \cong \mathrm{TSG}_{+}(\Gamma)$. Moreover, $\Gamma$ can be chosen to be a complete bipartite graph $K_{n, n}$ for some $n$.

We deduce from Theorems 2 and 3 that the set of orientation preserving topological symmetry groups of 3-connected embedded graphs in $S^{3}$ is exactly the set of finite subgroups of Diff $+\left(S^{3}\right)$. The finite subgroups of Diff $+\left(S^{3}\right)$ consist of the finite subgroups of $\mathrm{SO}(4)$, possibly together with the Milnor groups $Q(8 k, m, n)$ in the case where the subgroup acts freely on $S^{3}$ (see [DV] for the finite subgroups of $\mathrm{SO}(4)$, and [Mi] and [Z] for groups that could act freely on $S^{3}$ ). We note that Thurston's geometrization program [Th] would imply that the groups $Q(8 k, m, n)$ do not occur.

We prove Theorems 1 and 2 in Section 2, assuming two propositions which are proved in Sections 3 and 4 . The heart of the argument lies in the proof of Proposition 1 in Section 3, which uses the Characteristic Submanifold Theorem of Jaco-Shalen [JS] and Johannson [Jo] and Thurston's Hyperbolization Theorem [Th], in the context of pared manifolds. These results were applied in a similar fashion in [F2]. In the case of a 3-connected embedded graph $\Gamma$, the strategy is to re-embed $\Gamma$ in a "nicer" way as $\Delta$ such that $\operatorname{TSG}_{+}(\Gamma) \leq \operatorname{TSG}_{+}(\Delta)$ and $\operatorname{TSG}_{+}(\Delta)$ is induced by a finite subgroup of Diff $+\left(S^{3}\right)$. Finally, in Section 5, Theorem 3 is proved by a direct construction.

The first and fourth named authors first met and began collaborating on this project in the fall of 2000 during a visit to the Institut des Hautes Études Scientifiques. It is a pleasure to thank the Institut for its hospitality. The second author wishes to thank the California Institute of Technology for its hospitality during his sabbatical in the spring of 2002.

\section{Proofs of Theorems 1 and 2}

Let $\Gamma$ be a graph embedded in $S^{3}$ with underlying abstract graph $\gamma$. Recall that we defined $\operatorname{TSG}(\Gamma)$ as the subgroup of $\operatorname{Aut}(\gamma)$ induced by homeomorphisms of the pair $\left(S^{3}, \Gamma\right)$. However, for technical reasons we prefer to restrict our study to those homeomorphisms of $\left(S^{3}, \Gamma\right)$ which are diffeomorphisms except possibly on the set 
of vertices of $\Gamma$. By an abuse of terminology we shall call such a homeomorphism a diffeomorphism of $\left(S^{3}, \Gamma\right)$. Note that by standard smoothing theory (see [Moi]), if there is a homeomorphism of $\left(S^{3}, \Gamma\right)$ inducing an automorphism $\alpha$ of $\Gamma$, then there is also a diffeomorphism of $\left(S^{3}, \Gamma\right)$ inducing the automorphism $\alpha$. Thus we can equivalently define TSG $(\Gamma)$ as the subgroup of $\operatorname{Aut}(\gamma)$ induced by diffeomorphisms of $\left(S^{3}, \Gamma\right)$.

Let $G$ be a group of orientation preserving diffeomorphisms of $\left(S^{3}, \Gamma\right)$. Let $H$ be the image of $G$ under the natural homomorphism from $G$ to $\operatorname{TSG}_{+}(\Gamma)$. Then $H$ is said to be induced on $\Gamma$ by $G$.

A group $H$ is said to be realizable if there is an embedded graph $\Gamma$ with $\operatorname{TSG}_{+}(\Gamma) \cong$ $H$. In this case, we say that $H$ is realized by $\Gamma$.

We introduce some notation that we will use throughout the rest of the paper. Let $V$ denote the set of embedded vertices of an embedded graph $\Gamma$, and let $E$ denote the set of embedded edges of $\Gamma$. We shall construct a neighborhood $N(\Gamma)$ as the union of two sets, $N(V)$ and $N(E)$, which have disjoint interiors. For each vertex $v \in V$, let $N(v)$ denote a small ball around $v$, and let $N(V)$ denote the union of all of these balls. For each embedded edge $\varepsilon \in E$, let $N(\varepsilon)$ denote a tube $D^{2} \times I$ whose core is $\varepsilon-N(V)$, such that $N(\varepsilon)$ contains no other part of $\Gamma$, and $N(\varepsilon)$ meets $N(V)$ in a pair of disks. Let $N(E)$ denote the union of all the tubes $N(\varepsilon)$. Let $N(\Gamma)=N(V) \cup N(E)$. Throughout the paper we shall use $\partial^{\prime} N(\varepsilon)$ to denote the annulus $\partial N(\Gamma) \cap N(\varepsilon)$ in order to distinguish it from the sphere $\partial N(\varepsilon)$.

We will use $c l$ to denote the closure of a set and int to denote the interior of a set. Finally, by a chain of length $n$ we shall mean an arc in $\Gamma$ containing $n$ vertices of valence two and no vertices of higher valence in its interior such that neither endpoint of the arc has valence two. A single edge such that neither endpoint has valence two is said to be a chain of length zero.

We shall use spheres and pinched spheres to decompose $\Gamma$ into smaller pieces as follows.

Definition 1. Let $\Sigma$ be a 2 -sphere embedded in $S^{3}$. If $\Sigma$ intersects $\Gamma$ in a single vertex $v$ of valence more than two and each component of $S^{3}-\Sigma$ contains part of $\Gamma$, then we say that $\Sigma$ is a type I sphere and $v$ is a type I vertex of $\Gamma$. (See Figure 4.)

Observe that removing a type I vertex from $\Gamma$ separates $\Gamma$, but not every vertex which separates $\Gamma$ is a type I vertex.

Definition 2. Let $\Sigma$ be a 2 -sphere embedded in $S^{3}$. If $\Sigma$ intersects $\Gamma$ in vertices $v$ and $w$, the closure of neither component of $\left(S^{3}-\Sigma\right) \cap \Gamma$ is a single arc, and the annulus $\Sigma-\operatorname{int}(N(v) \cup N(w))$ is incompressible in $\operatorname{cl}\left(S^{3}-N(\Gamma)\right)$, then we say that $\Sigma$ is a type II sphere of $\Gamma$. (See Figure 5.) 


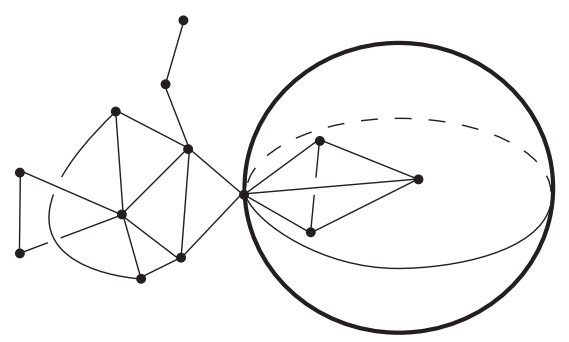

Figure 4. An embedded graph with a type I sphere

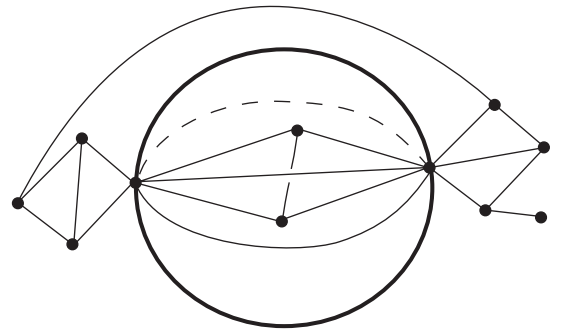

Figure 5. An embedded graph with a type II sphere

Definition 3. Let $\Sigma$ be a 2-sphere with two points identified to a single point $p$. We say that $\Sigma$ is a pinched sphere and $p$ is the pinch point. Let $\Sigma$ be a pinched sphere in $S^{3}$, with pinch point $p$. Suppose that $p$ is a vertex of $\Gamma$ such that $\Sigma \cap \Gamma=\{p\}$, each component of $S^{3}-\Sigma$ contains part of $\Gamma$, and the annulus $\Sigma-\operatorname{int}(N(p))$ is incompressible in $\operatorname{cl}\left(S^{3}-N(\Gamma)\right.$ ). Then we say that $\Sigma$ is a type III sphere of $\Gamma$. (See Figure 6.)

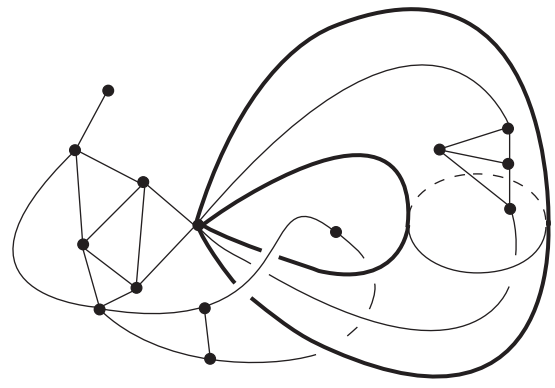

Figure 6. An embedded graph with a type III sphere

We remark that our definition of a type I sphere is close to that of Suzuki [Su], however, our definition of a type II sphere is different from his. 
We will use the following two propositions to prove Theorems 1 and 2. The proofs of these propositions will be given in the next two sections.

Proposition 1. Let $\Gamma$ be an embedded graph with no type I spheres and let $H=$ $\mathrm{TSG}_{+}(\Gamma)$. Then either $H$ is isomorphic to a finite subgroup of $\operatorname{Diff}_{+}\left(S^{3}\right), H \cong S_{r}$ for some $r$, or $H$ has a non-trivial normal subgroup $N$ such that both $N$ and $H / N$ are realizable. Furthermore, if $\Gamma$ has no type II or type III spheres, then $\Gamma$ can be reembedded as $\Delta$ such that $H \leq \mathrm{TSG}_{+}(\Delta)$, and $H$ is induced on $\Delta$ by an isomorphic finite subgroup of $\operatorname{Diff}_{+}\left(S^{3}\right)$.

If $H \cong S_{r}$ and $H$ is simple, then $H \cong \mathbb{Z}_{2}$. Thus it follows from Proposition 1 that if $\Gamma$ has no type I spheres and $\operatorname{TSG}_{+}(\Gamma)$ is a simple group then $\operatorname{TSG}_{+}(\Gamma)$ is isomorphic to a finite subgroup of $\operatorname{Diff}_{+}\left(S^{3}\right)$.

Proposition 2. Let $\Gamma$ be an embedded graph and let $H=\mathrm{TSG}_{+}(\Gamma)$. Then either $H$ is realized by a graph with no type I spheres, $H \cong S_{r}$ for some $r$, or $H$ has a non-trivial normal subgroup $N$ such that both $N$ and $H / N$ are realizable.

If $H \cong \mathbb{Z}_{2}$, then $H$ is realized by the graph consisting of a single edge. Hence it follows from Proposition 2 that any realizable simple group can be realized by an embedded graph with no type I spheres.

We now prove Theorems 1 and 2 from these propositions.

Theorem 1. Let $\Gamma$ be an embedded graph.

a) If $\mathrm{TSG}_{+}(\Gamma)$ is a simple group, then it is either the alternating group $A_{5}$ or a cyclic group of prime order.

b) In general, the sequence of quotient groups in any composition series for $\mathrm{TSG}_{+}(\Gamma)$ contains only alternating groups $A_{n}$ with $n \geqslant 5$ and cyclic groups.

Proof. To prove part (a), we observe that by Proposition 2, there is an embedded graph $\Delta$ with no type I spheres such that $\operatorname{TSG}_{+}(\Gamma) \cong \mathrm{TSG}_{+}(\Delta)$. Then by Proposition 1 , $\mathrm{TSG}_{+}(\Delta)$ is isomorphic to a finite subgroup of Diff $+\left(S^{3}\right)$. However, it is shown in [Z, Theorem 1] that the alternating group $A_{5}$ is the only non-abelian finite simple group which acts faithfully by diffeomorphisms on a homology 3 -sphere. The result follows.

To prove part (b), we use induction on the number of elements in $H=\mathrm{TSG}_{+}(\Gamma)$. Let $|H|=k$ and assume the result is true for all realizable groups with fewer than $k$ elements. If $H$ is simple, then we are done by part (a). Otherwise Propositions 1 and 2 imply that either $H$ is a finite subgroup of $\operatorname{Diff}_{+}\left(S^{3}\right), H \cong S_{r}$ for some $r$, or $H$ has a non-trivial normal subgroup $N$ such that both $N$ and $H / N$ are realizable. In the first case, the result follows from [Z, Theorem 2], while the second case is clear. 
In the third case, we know by induction that both $N$ and $H / N$ have composition series all of whose simple quotients are either alternating or cyclic. Putting these two series together gives a composition series for $H$ with the same property. Finally, the Jordan-Hölder theorem implies that this also holds for any other composition series for $H$.

By a similar argument, we can prove that for any realizable group $H$, the number of quotients in a composition series for $H$ which are isomorphic to $\mathbb{Z}_{2}$ is at least as large as the number of quotients which are isomorphic to any $A_{r}$ with $r \geqslant 6$. To see this, observe that if $H$ is a finite subgroup of $\operatorname{Diff}_{+}\left(S^{3}\right)$ or $H \cong S_{r}$, then the result follows; otherwise, the proof uses induction on the order of $H$, as above. A complete characterization of all realizable groups may be possible, working along the lines of [B2].

According to [B2, Corollary 9.15], the group $G=A_{5} \times \mathbb{Z}_{3}$ is not the automorphism group of any planar graph. However, we see as follows that $G$ is realizable. It follows from [M] that $A_{5}$ is the automorphism group of a 3-connected planar graph which can be realized as the 1 -skeleton $X$ of a convex polytope $P$ in $\mathbb{R}^{3}$, such that all abstract automorphisms of $X$ are induced by isometries of $P$. Let $\Gamma_{1}$ be obtained from $X$ by connecting each vertex of $X$ to a point $v_{1}$ in the interior of $P$. Now let $\Gamma_{2}$ be the 1-skeleton of a tetrahedron disjoint from $P$. Tie the same non-invertible knot in each of the three edges of $\Gamma_{2}$ which do not contain a particular vertex $v_{2}$, all oriented in the same way. Finally, form $\Gamma$ from $\Gamma_{1}$ and $\Gamma_{2}$ by connecting $v_{1}$ to $v_{2}$ by an arc which does not meet the rest of $\Gamma_{1}$ or $\Gamma_{2}$. Then $\mathrm{TSG}_{+}(\Gamma)=A_{5} \times \mathbb{Z}_{3}$. Thus there exist realizable groups which are not the automorphism group of any planar graph.

Theorem 2. Let $\Gamma$ be an embedded 3-connected graph. Then $\mathrm{TSG}_{+}(\Gamma)$ is isomorphic to a finite subgroup of $\operatorname{Diff}_{+}\left(S^{3}\right)$.

Proof. If $\Gamma$ has a type I or III sphere then $\Gamma$ can be disconnected be removing a single vertex and the edges incident to it. If $\Gamma$ has a type II sphere then $\Gamma$ can be disconnected by removing two vertices and the edges incident to them. Thus if $\Gamma$ is 3-connected, then $\Gamma$ has no type I, II, or III spheres. So the result follows by Proposition 1 .

We also use Proposition 1 to prove the following strengthening of Theorem 2.

Proposition 3. Let $H=\mathrm{TSG}_{+}(\Gamma)$ for some embedded 3-connected graph $\Gamma$. Then $\Gamma$ can be re-embedded as $\Delta$ such that $H$ is a subgroup of $\mathrm{TSG}_{+}(\Delta)$ and $\mathrm{TSG}_{+}(\Delta)$ is induced by an isomorphic finite subgroup of $\operatorname{Diff}_{+}\left(S^{3}\right)$.

Proof. Let $H_{1}=\mathrm{TSG}_{+}(\Gamma)$. Then, as in the proof of Theorem 2, $\Gamma$ has no type I, II or III spheres. Hence by Proposition 1 we can re-embed $\Gamma$ as $\Gamma_{1}$ such that $H_{1} \leq \mathrm{TSG}_{+}\left(\Gamma_{1}\right)$, and $H_{1}$ is induced by an isomorphic subgroup $K_{1}$ of Diff $+\left(S^{3}\right)$. If 
$\mathrm{TSG}_{+}\left(\Gamma_{1}\right)=H_{1}$, then we are done by letting $\Delta=\Gamma_{1}$. If $H_{1}$ is a proper subgroup of $\mathrm{TSG}_{+}\left(\Gamma_{1}\right)$, we let $H_{2}=\mathrm{TSG}_{+}\left(\Gamma_{1}\right)$ and again apply Proposition 1 to the 3-connected embedded graph $\Gamma_{1}$. Continue this process. Then for each $i, \mathrm{TSG}_{+}\left(\Gamma_{i}\right)$ is a subgroup of $\operatorname{Aut}(\gamma)$, where $\gamma$ is the underlying abstract graph of $\Gamma$. Since Aut $(\gamma)$ is finite, this process cannot go on indefinitely.

\section{Proof of Proposition 1}

We assume the reader is familiar with standard 3-manifold topology. However, we will need to use some terminology and results about pared manifolds which we give below.

Definition 4. A pared 3-manifold $(M, P)$ is an orientable 3-manifold $M$ together with a family $P$ of disjoint incompressible annuli and tori in $\partial M$.

A pared manifold is a special case of a manifold with boundary patterns in the sense of Johannson [Jo] or a 3-manifold pair in the sense of Jaco-Shalen [JS]. The following definitions agree with those of [Jo] and [JS].

Definition 5. A pared manifold $(M, P)$ is said to be simple if it satisfies the following three conditions:

1) $M$ is irreducible and $\partial M-P$ is incompressible.

2) Every incompressible torus in $M$ is parallel to a torus component of $P$.

3) Any annulus $A$ in $M$ with $\partial A$ contained in $\partial M-P$ is either compressible or parallel to an annulus $A^{\prime}$ in $\partial M$ with $\partial A^{\prime}=\partial A$ and such that $A^{\prime} \cap P$ consists of zero or one annular component of $P$.

Definition 6. A pared manifold $(M, P)$ is said to be Seifert fibered if there is a Seifert fibration of $M$ for which $P$ is a union of fibers. A pared manifold $(M, P)$ is said to be $I$-fibered if there is an $I$-bundle map of $M$ over a surface $B$ such that $P$ is the preimage of $\partial B$.

We will use the following results about pared manifolds.

Characteristic Submanifold Theorem for Pared Manifolds ([JS] and [Jo]). Let $(X, P)$ be a pared manifold with $X$ irreducible and $\partial X-P$ incompressible. Then, up to an isotopy of $(X, P)$, there is a unique family $\Omega$ of disjoint incompressible tori and annuli with $\partial \Omega$ contained in $\partial X-P$ such that the following two conditions hold:

1) If $W$ is the closure of a component of $X-\Omega$, then the pared manifold ( $W, W \cap$ $(P \cup \Omega))$ is either simple, Seifert fibered, or I-fibered. 
2) There is no family $\Omega^{\prime}$ with fewer elements than $\Omega$ which satisfies the above.

Thurston's Theorem for Pared Manifolds ([Th]). If $(M, P)$ is simple, $M$ is connected, and $\partial M$ is non-empty, then either $M-P$ admits a finite volume complete hyperbolic metric with totally geodesic boundary, or $(M, P)$ is Seifert fibered or I-fibered.

Now we are ready to prove Proposition 1. Since the proof of Proposition 1 is long, we begin with an outline. In Step 1, we will follow the proof of [F2, Theorem 1], and use the Characteristic Submanifold Theorem ([JS] and [Jo]) to split the complement of $N(\Gamma)$ along a minimal family $\Theta$ of incompressible tori which is unique up to ambient isotopy and such that each component is either simple or Seifert fibered. We let $X$ denote the component which contains $\partial N(\Gamma)$. Since $\Gamma$ has no type I spheres, we can then use the Characteristic Submanifold Theorem for Pared Manifolds to split $X$ along a minimal family $\Omega$ of incompressible annuli which is unique up to ambient isotopy and such that as a pared manifold each component is either simple, Seifert fibered, or I-fibered. We then define a group $G$ of orientation preserving diffeomorphisms of $\left(S^{3}, \Gamma\right)$ such that for every $g \in G, g(\Theta)=\Theta$ and $g(\Omega)=\Omega$, and every $a \in \mathrm{TSG}_{+}(\Gamma)$ is induced by some $g_{a} \in G$.

In Step 2, we choose a particular component $W$ of $X-\Omega$ which is setwise invariant under $G$, such that $G$ permutes some of the elements of $\Omega$ that are contained in $\partial W$ or some of the components of $\partial N(V) \cap W$. In Step 3, we show that the proof can be reduced to analyzing the action that $G$ induces on $W$. In Steps 4,5 , and 6 we obtain our result in the cases where $W$ is Seifert fibered, I-fibered, and simple, respectively.

Proof of Proposition 1. Let $\Gamma$ be an embedded graph with no type I spheres, and let $\gamma$ denote the underlying abstract graph of $\Gamma$. The result is clear if $\mathrm{TSG}_{+}(\Gamma)$ is trivial. So we assume it is non-trivial. We begin by considering the special cases where $\gamma$ is homeomorphic to an arc or a circle. In these cases, the full automorphism group $\operatorname{Aut}(\gamma)$ is either a finite cyclic group or a dihedral group, and hence $\operatorname{TSG}_{+}(\Gamma)$ is a finite subgroup of $\operatorname{Diff}_{+}\left(S^{3}\right)$. We embed $\Delta$ as an embedding of $\gamma$ which is a planar round circle or a line segment. Then $\operatorname{TSG}_{+}(\Delta)=\operatorname{Aut}(\gamma)$, and there is a subgroup $G$ of Diff $+\left(S^{3}\right)$ such that $G \cong H$ and $G$ induces $H$ on $\Delta$.

From now on, we assume that $\gamma$ is not a simple closed curve or an arc. Hence $\Gamma$ has some vertex with valence at least three. Also since $\Gamma$ has no type I spheres, $\Gamma$ cannot have any vertices of valence one. Let $m$ be a number larger than the total number of vertices in $\Gamma$. We will use $m$ at several places in the proof.

Step 1. We split the complement of $\Gamma$ along characteristic tori and annuli.

Let $M=\operatorname{cl}\left(S^{3}-N(\Gamma)\right)$. Since $\Gamma$ is a connected graph, $M$ is irreducible. So we can apply the Characteristic Submanifold Theorem to $M$ to get a minimal family of incompressible tori, $\Theta$, in $M$ such that the closure of every component of $M-\Theta$ is 
either simple or Seifert fibered, and $\Theta$ is unique up to an isotopy of $M$ fixing $\partial M$ pointwise. It follows from the uniqueness of $\Theta$ and $N(\Gamma)$ up to isotopy that for every automorphism $a \in \mathrm{TSG}_{+}(\Gamma)$, there is an orientation preserving diffeomorphism $g:\left(S^{3}, \Gamma\right) \rightarrow\left(S^{3}, \Gamma\right)$ which induces $a$, such that $g(\Theta)=\Theta, g(N(V))=N(V)$, and $g(N(E))=N(E)$. Since $\partial M$ is connected, there is a unique component of $M-\Theta$ which contains $\partial M$. Let $X$ denote the closure of this component. Then for each diffeomorphism $g:\left(S^{3}, \Gamma\right) \rightarrow\left(S^{3}, \Gamma\right)$ such that $g(M)=M$ and $g(\Theta)=\Theta$, we have $g(X)=X$. If $X$ is Seifert fibered, then $\partial X$ is a collection of tori, and hence $\Gamma$ is a simple closed curve, contrary to our assumption above. Thus $X$ must be simple. So every incompressible torus in $X$ is boundary parallel. Also note that since each torus boundary component of $X$ is incompressible in $M$ and $M$ is irreducible, $X$ must be irreducible.

Now $\partial X$ consists of $\partial N(\Gamma)$ together with a collection of tori in $\Theta$. Let $P$ denote the union of the annuli in $\partial^{\prime} N(E)$ and the torus boundary components of $X$. Observe that $\operatorname{cl}(\partial X-P)=\partial N(V) \cap \partial X$ consists of a collection of spheres with holes.

We show as follows that $\partial X-P$ is incompressible in $X$. Suppose that there is a non-trivial loop $L$ in some component of $\partial X-P$ which compresses in $X$. Then $L$ is contained in some $\partial N(v) \cap \partial X$ and $L$ bounds a disk $D_{1}$ in $X$. Also $L$ bounds a disk $D_{2}$ in $N(v)$ such that $D_{2}$ intersects $\Gamma$ only in $v$. Now $\Sigma=D_{1} \cup D_{2}$ is a sphere, whose intersection with $\Gamma$ is the vertex $v$. Since $L$ is non-trivial in $\partial N(v) \cap \partial X$, each component of $S^{3}-\Sigma$ contains part of $\Gamma$. Recall that $\Gamma$ has no vertices of valence one. If the valence of $v$ is two, we can slide $\Sigma$ along an $\operatorname{arc}$ in $\Gamma$ until $\Sigma$ intersects $\Gamma$ at a vertex with valence at least three. But this gives us a type I sphere for $\Gamma$, contrary to hypothesis. Hence $\partial X-P$ must be incompressible in $X$.

Since $X$ is irreducible and $\partial X-P$ is incompressible in $X$, we can now apply the Characteristic Submanifold Theorem for Pared Manifolds to the pair $(X, P)$. This gives us a minimal family $\Omega$ of incompressible tori and annuli in $X$ with the boundary of each component of $\Omega$ contained in $\partial X-P$, such that if $W$ is the closure of any component of $X-\Omega$, then the pared manifold $(W, W \cap(P \cup \Omega)$ ) is either simple, Seifert fibered, or $I$-fibered, and $\Omega$ is unique up to an isotopy of $(X, P)$. Since every incompressible torus in $X$ is boundary parallel, and the family $\Omega$ is minimal, $\Omega$ cannot contain any tori. Thus $\Omega$ is a (possibly empty) family of incompressible annuli in $X$. Furthermore, for any $W, \partial W-(W \cap(P \cup \Omega)) \subset \partial X-P$ and $W \subset X$. Thus since $\partial X-P$ is incompressible in $X, \partial W-(W \cap(P \cup \Omega))$ is incompressible in $W$.

We denote by $G$ the group of all those orientation preserving diffeomorphisms $g:\left(S^{3}, \Gamma\right) \rightarrow\left(S^{3}, \Gamma\right)$ for which $g(N(V))=N(V), g(N(E))=N(E), g(\Theta)=\Theta$ and $g(\Omega)=\Omega$. Then $P$ and $X$ are each setwise invariant under $G$. Also by the uniqueness of each of the sets $N(V), N(E)$, and $\Theta$, up to an isotopy of $\left(S^{3}, \Gamma\right)$ and the uniqueness of $\Omega$ up to an isotopy of $(X, P)$, it follows that every $a \in \mathrm{TSG}_{+}(\Gamma)$ is induced by some $g_{a} \in G$. 
For each annulus $A$ in $\Omega$, the boundary of $A$ is contained in $\partial X-P$. So each component of $\partial A$ is contained in some $\partial N(v)$. Thus each component of $\partial A$ bounds a disk $D$ in some $N(v)$ such that $D \cap \Gamma=\{v\}$. Furthermore, we can choose the collection of these disks to be pairwise disjoint except possibly on the set of vertices $V$. Thus for each $A \in \Omega$, there is a pair of disks $D_{1}$ and $D_{2}$ such that $\Sigma=A \cup D_{1} \cup D_{2}$ is either a sphere meeting $\Gamma$ in two vertices or a pinched sphere with its pinch point at a vertex. Let $\Lambda$ denote the collection of these spheres and pinched spheres. Then the elements of $\Lambda$ are pairwise disjoint except possibly on $V$. Since the collection of these disks is unique up to an isotopy of $N(V)$ fixing both $\Gamma$ and $\partial N(V)$, we can assume that we chose $G$ such that for every $g \in G, g(\Lambda)=\Lambda$.

Since every $A \in \Omega$ is incompressible in $X$, and every torus component of $\partial X$ is incompressible in $M$, every $A \in \Omega$ is incompressible in $M$. Thus if $\Sigma \in \Lambda$ is a sphere and the closure of neither component of $\left(S^{3}-\Sigma\right) \cap \Gamma$ is a single arc, then $\Sigma$ is a type II sphere; and if $\Sigma \in \Lambda$ is a pinched sphere and the closure of neither component of $\left(S^{3}-\Sigma\right) \cap \Gamma$ is a single vertex, then $\Sigma$ is a type III sphere.

Step 2. We choose a setwise invariant component $W$ of $X-\Omega$ such that $G$ permutes some of the elements of $\Omega$ in $\partial W$ or some of the components of $\partial N(V) \cap W$.

If $\Omega$ is empty, let $W=X$. Then every component of $\partial N(V)$ meets $W$, and hence $G$ permutes some of the components of $\partial N(V) \cap W$. So we suppose that $\Omega$ is non-empty.

The proof of [F2, Theorem 1] shows that if $\Gamma$ is a 3-connected graph then there is a unique component of $X-\Omega$ whose closure $W$ has the properties that: every element of $\Omega$ is contained in $\partial W$ and for every $\Sigma_{i} \in \Lambda$ the closure of the component of $S^{3}-\Sigma_{i}$ which is disjoint from $W$ meets $\Gamma$ in an arc if $\Sigma_{i}$ is a sphere and in a single vertex if $\Sigma_{i}$ is a pinched sphere. The proof that there is such a $W$ is analogous if we replace the hypothesis that $\Gamma$ is 3 -connected by the hypothesis that $\Gamma$ has no type I, II, or III spheres. Thus if $\Gamma$ has no type I, II or III spheres, then we choose this $W$. By the uniqueness of $W$ we know that $W$ is setwise invariant under $G$. Also if $v$ is any vertex with valence at least three, then $\partial N(v) \cap W$ contains a sphere with at least three holes. Hence for every non-trivial $a \in \mathrm{TSG}_{+}(\Gamma), g_{a}$ induces a non-trivial permutation of either the elements of $\Omega$ or the components of $\partial N(V) \cap W$. Thus we are done with Step 2 in the case where $\Gamma$ has no type II or III spheres.

In order to choose $W$ when $\Gamma$ does have a type II or type III sphere, we will first associate an abstract graph $\lambda$ with the set $\Lambda$ of spheres and pinched spheres. For each component $Y$ of $S^{3}-\Lambda$, let $y$ be a vertex in $\lambda$; and for every pair of components $Y$ and $Z$ of $S^{3}-\Lambda$, let there be an edge in $\lambda$ between the vertices $y$ and $z$ if and only if there is some $\Sigma \in \Lambda$ which is contained in the boundary of both $Y$ and $Z$. Observe that because every element of $\Lambda$ separates $S^{3}$, the graph $\lambda$ is a tree. Since $G$ takes $\Lambda$ to itself, every $g \in G$ defines an automorphism $g^{\prime}$ of $\lambda$. Let $G^{\prime}$ be the group of automorphisms of $\lambda$ induced by $G$. Since $\lambda$ is a tree, it follows from elementary 
graph theory that there is either a vertex or an edge of $\lambda$ that is invariant under $G^{\prime}$. If no vertex of $\lambda$ is fixed by $G^{\prime}$, then there is an invariant edge $e$ of $\lambda$ whose vertices are interchanged by some element of $G^{\prime}$. In this case, there is a sphere $\Sigma \in \Lambda$ which is invariant under $G$, and some element $g \in G$ which interchanges the two components of $S^{3}-\Sigma$. We handle this case as follows.

Claim 1. Suppose that there is a sphere $\Sigma \in \Lambda$ which is invariant under $G$, and some $g \in G$ which interchanges the components of $S^{3}-\Sigma$. Then either $H \cong \mathbb{Z}_{2}$ or $H$ has a non-trivial normal subgroup $N$ such that both $N$ and $H / N$ are realizable.

Proof of Claim 1. The closures of the components of $S^{3}-\Sigma$ intersect $\Gamma$ in subgraphs $\alpha_{1}$ and $\alpha_{2}$. Since $\Sigma$ is invariant under $G$, we can define a homomorphism $\Phi: \mathrm{TSG}_{+}(\Gamma) \rightarrow \mathbb{Z}_{2}$ as follows. For each $a \in \mathrm{TSG}_{+}(\Gamma)$, let $\Phi(a)=0$ if $a$ takes each $\alpha_{i}$ to itself, and let $\Phi(a)=1$ if $a$ interchanges $\alpha_{1}$ and $\alpha_{2}$. Since some $a \in \mathrm{TSG}_{+}(\Gamma)$ interchanges $\alpha_{1}$ and $\alpha_{2}$, $\Phi$ must be onto.

If $\operatorname{ker}(\Phi)$ is trivial then $\operatorname{TSG}_{+}(\Gamma) \cong \mathbb{Z}_{2}$. So we assume $N=\operatorname{ker}(\Phi)$ is nontrivial. We create a new embedded graph by adding a vertex in the interior of every edge in $\alpha_{1}$ which meets $\Sigma$, and adding an edge incident to each of these new vertices. Let $\Pi$ denote $\Gamma$ with these new vertices and edges. Then every $a^{\prime} \in \mathrm{TSG}_{+}(\Pi)$ takes this set of new edges to itself and hence induces some $a \in \mathrm{TSG}_{+}(\Gamma)$ which does not interchange $\alpha_{1}$ and $\alpha_{2}$. Also every $a \in \operatorname{ker}(\Phi)$ naturally defines an $a^{\prime} \in \mathrm{TSG}_{+}(\Pi)$. It is thus easy to see that $\mathrm{TSG}_{+}(\Pi) \cong \operatorname{ker}(\Phi)$. Finally, let $\Pi^{\prime}$ denote the graph consisting of a single edge, then $\mathrm{TSG}_{+}\left(\Pi^{\prime}\right) \cong \mathbb{Z}_{2} \cong H / N$. Now $N$ and $H / N$ are both realizable. This proves Claim 1 .

Because of Claim 1, we assume no such sphere $\Sigma$ exists. Hence there is some vertex of $\lambda$ which is invariant under $G^{\prime}$. First suppose that $G^{\prime}$ does not act trivially on $\lambda$. Then there is some vertex $x$ of $\lambda$ which is fixed by $G^{\prime}$ and which is adjacent to a vertex of $\lambda$ which is not fixed by $G^{\prime}$. In this case, we choose $W$ to be the closure of the component of $X-\Omega$ which corresponds to the vertex $x$ of $\lambda$. Then $W$ will be setwise invariant under $G$, however some element of $\Omega$ which is contained in $\partial W$ is not setwise invariant under $G$. Finally, suppose that $G^{\prime}$ acts trivially on $\lambda$. Then every component of $X-\Omega$ is setwise invariant under $G$. Since $\operatorname{TSG}_{+}(\Gamma)$ is not trivial, there is some vertex $v$ of $\Gamma$ which is not fixed by $G$. Let $W$ be the closure of some component of $X-\Omega$ which meets $\partial N(v)$. Thus we are done with Step 2.

Before we begin Step 3, we introduce some notation. Let $A_{1}, \ldots, A_{n}$ denote those annuli in $\Omega$ which are contained in $\partial W$, and let $\Sigma_{1}, \ldots, \Sigma_{n}$ denote the spheres or pinched spheres of $\Lambda$ containing $A_{1}, \ldots, A_{n}$ respectively. If $\Sigma_{i}$ is a sphere, let $\Sigma_{i} \cap \Gamma=\left\{v_{i}, w_{i}\right\}$, and if $\Sigma_{i}$ is a pinched sphere, let $\Sigma_{i} \cap \Gamma=\left\{u_{i}\right\}$. For each $i$, let $c_{i}$ and $d_{i}$ denote the boundary components of $A_{i}$, such that if $\Sigma_{i}$ is a sphere $c_{i} \subset \partial N\left(v_{i}\right)$ and $d_{i} \subset \partial N\left(w_{i}\right)$, and if $\Sigma_{i}$ is a pinched sphere $c_{i} \cup d_{i} \subset \partial N\left(u_{i}\right)$. For each $\Sigma_{i}$, we let $B_{i}$ denote the closure of the component of $S^{3}-\Sigma_{i}$ whose interior 
is disjoint from $W$ and let $\Gamma_{i}=B_{i} \cap \Gamma$. The sets $\left\{\Gamma_{1}, \ldots, \Gamma_{n}\right\}$ and $\left\{B_{1}, \ldots, B_{n}\right\}$ are each setwise invariant under $G$, since $W$ is setwise invariant under $G$. It follows that $\Gamma^{\prime}=\operatorname{cl}\left(\Gamma-\left(\Gamma_{1} \cup \cdots \cup \Gamma_{n}\right)\right)$ is setwise invariant under $G$. (Note that $\Gamma^{\prime}$ is not necessarily connected.)

Step 3. We reduce the proof to analyzing the action that $G$ induces on $W$.

In particular, we prove the following.

Claim 2. Suppose that there is some $g \in G$ and $A_{i} \in \Omega$ such that $g\left(A_{i}\right)=A_{i}$, $g\left(c_{i}\right)=c_{i}, g\left(d_{i}\right)=d_{i}$, and $g$ induces a non-trivial automorphism on $\Gamma_{i}$. Then $\Gamma$ has a type II or type III sphere and $H$ has a non-trivial normal subgroup $N$ such that both $N$ and $H / N$ are realizable.

Proof of Claim 2. Observe that $\Gamma_{i}$ is neither an arc nor a single vertex, since otherwise $g$ cannot induce a non-trivial automorphism on $\Gamma_{i}$. Since $G$ permutes some elements of $\Omega$ in $\partial W$ or some components of $\partial N(V) \cap W, \operatorname{cl}\left(\Gamma-\Gamma_{i}\right)$ is neither an arc nor a single vertex. Thus $\Gamma$ has a type II or type III sphere.

Before we define $N$, we use $g$ to create another orientation preserving diffeomorphism $h$ of $\left(S^{3}, \Gamma\right)$ as follows. First we let $h\left|B_{i}=g\right| B_{i}$. Then $h\left(B_{i}\right)=B_{i}, h \mid B_{i}$ is orientation preserving, $h\left(c_{i}\right)=c_{i}$, and $h\left(d_{i}\right)=d_{i}$. It follows that $h \mid \Sigma_{i}$ is isotopic to the identity on $\Sigma_{i}$ by an isotopy fixing $v_{i}$ and $w_{i}$ if $\Sigma_{i}$ is a sphere or fixing $u_{i}$ if $\Sigma_{i}$ is a pinched sphere. Let $C_{i}$ be a ball or a pinched ball (according to which $B_{i}$ is) such that $C_{i} \cap \Gamma=\Gamma_{i}$ and respectively $B_{i}-\left\{v_{i}, w_{i}\right\} \subset \operatorname{int}\left(C_{i}\right)$ or $B_{i}-\left\{u_{i}\right\} \subset \operatorname{int}\left(C_{i}\right)$. Extend $h$ to a diffeomorphism of $C_{i}-B_{i}$ such that $h$ is the identity on $\partial C_{i}$. Then extend $h$ to the rest of $S^{3}$ by the identity. Now let $a \in \mathrm{TSG}_{+}(\Gamma)$ be induced by $h$. Then $a \mid \operatorname{cl}\left(\Gamma-\Gamma_{i}\right)$ is the identity and $g$ induces $a$ on $\Gamma_{i}$. By hypothesis $a \mid \Gamma_{i}$ is non-trivial.

Let $N$ be the set of all $\alpha \in \mathrm{TSG}_{+}(\Gamma)$ such that $\alpha \mid \Gamma^{\prime}$ is the identity, and for each $j \leqslant n, \alpha\left(\Gamma_{j}\right)=\Gamma_{j}$ and $g_{\alpha}\left(c_{j}\right)=c_{j}$ and $g_{\alpha}\left(d_{j}\right)=d_{j}$. Then $N$ is a normal subgroup of $H$ containing the non-trivial element $a$. Observe that for each $\alpha \in N$, $g_{\alpha}$ does not permute any of the $A_{j}$ 's or any of the components of $\partial N(V) \cap W$. Thus by our choice of $W, N \neq \mathrm{TSG}_{+}(\Gamma)$. We shall prove that $N$ and $H / N$ are both realizable by constructing embedded graphs $\Pi$ and $\Pi^{\prime}$ with $\mathrm{TSG}_{+}(\Pi) \cong N$ and $\mathrm{TSG}_{+}\left(\Pi^{\prime}\right) \cong H / N$.

First we construct $\Pi$. We may assume that there is some $q \geq 1$ such that $\Sigma_{j}$ is a type II or type III sphere if and only if $j \leq q$. For each $j$ such that $\Sigma_{j}$ is a type III sphere, let $\varepsilon_{j}$ be an edge of $\Gamma_{j}$ which meets $\Gamma^{\prime}$. Now for each edge $\varepsilon$ in the orbit of $\varepsilon_{j}$ under $N$, we add a vertex to int $(\varepsilon)$ and an edge incident to this new vertex. For each such $j$, we let $\Gamma_{j}^{\prime}$ denote $\Gamma_{j}$ with this collection of vertices and edges added. For each $j$ such that $\Sigma_{j}$ is a type II sphere, if $\Gamma_{j}$ is not connected, let $\Gamma_{j}^{\prime}$ denote $\Gamma_{j}$ together with a new edge $e_{j} \subset \Sigma_{j}$ with vertices $v_{j}$ and $w_{j}$. For all other $\Gamma_{j}$, we let $\Gamma_{j}^{\prime}=\Gamma_{j}$. 
We obtain $\Pi$ by stringing together $\Gamma_{1}^{\prime}, \ldots, \Gamma_{n}^{\prime}$ with $\operatorname{arcs} \beta_{1}, \ldots, \beta_{n+1}$ as follows. For each $j$ such that $1 \leq j \leq n+1$, let $\beta_{j}$ denote an arc containing $j m$ vertices in its interior. We add $\beta_{1}, \ldots, \beta_{n+1}$ to $\Gamma_{1}^{\prime} \cup \ldots \cup \Gamma_{r}^{\prime}$ on the outside of $B_{1}, \ldots, B_{n}$, such that one endpoint of $\beta_{1}$ is attached to $\Gamma_{1}^{\prime}$ at the vertex $w_{1}$ or $u_{1}$; for each $j$ such that $1<j<n+1, \beta_{j}$ has one endpoint at $v_{j}$ or $u_{j}$ and the other endpoint at $w_{j+1}$ or $u_{j+1}$; and one endpoint of $\beta_{n+1}$ is attached to $\Gamma_{n}^{\prime}$ at $v_{n}$ or $u_{n}$. For each $j \leq q, \beta_{j}$ is the only chain in $\Pi$ of length $j m$, and hence each $\beta_{j}$ is invariant under $\operatorname{TSG}_{+}(\Pi)$. This implies that each $\Gamma_{j}^{\prime}$ is also setwise invariant under $\operatorname{TSG}_{+}(\Pi)$. It can be shown that $\mathrm{TSG}_{+}(\Pi)$ is induced by a group of diffeomorphisms of $\left(S^{3}, \Pi\right)$ which leave each $A_{j}$ setwise invariant. Furthermore, because of the additional edges that we added to those $\Gamma_{k}$ where $\Sigma_{k}$ is a pinched sphere, no diffeomorphism of $\left(S^{3}, \Pi\right)$ interchanges the boundary components of any $A_{j}$. Thus it is not hard to show that $N \cong \mathrm{TSG}_{+}(\Pi)$, and hence $N$ is realizable.

Next we construct an embedded graph $\Pi^{\prime}$ such that $\operatorname{TSG}_{+}\left(\Pi^{\prime}\right) \cong H / N$. Without loss of generality there are natural numbers $r$ and $s$ with $s \leq r \leq n$ such that $\left\{\Gamma_{1}, \ldots, \Gamma_{r}\right\}$ consists of one representative from each orbit of the $\Gamma_{i}$ under $\operatorname{TSG}_{+}(\Gamma)$, and there is some $g \in G$ which interchanges the boundary components of $A_{j}$ if and only if $j \leqslant s$. For each $j \leq s$, let $\beta_{j}$ denote an arc with $j m+2$ vertices in its interior, and let $x_{j}$ and $y_{j}$ denote the valence 2 vertices of $\beta_{j}$ which are adjacent to the endpoints. Thus there are $j m$ vertices of valence 2 on $\beta_{j}$ between $x_{j}$ and $y_{j}$. For each $j \leq s$, let $\beta_{j}^{\prime}$ denote $\beta_{j}$ with single edges attached at $x_{j}$ and at $y_{j}$. For each $j$ such that $s<j \leq r$, let $\beta_{j}$ denote an arc with $j m+1$ vertices in its interior, and let $x_{j}$ be a valence 2 vertex which is adjacent to one endpoint of $\beta_{j}$. Thus there are $j m$ vertices on $\beta_{j}$ between $x_{j}$ and the other endpoint of $\beta_{j}$. For each $j>s$, let $\beta_{j}^{\prime}$ denote $\beta_{j}$ with a single edge attached at $x_{j}$. Observe that there is an automorphism of $\beta_{j}^{\prime}$ which interchanges the endpoints of the $\operatorname{arc} \beta_{j}$ if and only if $j \leq s$. Also no $\beta_{j}^{\prime}$ has a non-trivial automorphism which fixes both endpoints of the $\operatorname{arc} \beta_{j}$. Now for each $k \leq n$, there is a $j \leq r$ such that $\Gamma_{k}$ is in the orbit of $\Gamma_{j}$ under $\operatorname{TSG}_{+}(\Gamma)$. For each such $k$, let $\beta_{k}^{\prime}$ be a copy of $\beta_{j}^{\prime}$, and let $x_{k}$ and $y_{k}$ be those vertices of $\beta_{k}^{\prime}$ corresponding to the vertices $x_{j}$ and $y_{j}$ of $\beta_{j}^{\prime}$.

We obtain $\Pi^{\prime}$ from $\Gamma^{\prime}$ by adding $\beta_{1}^{\prime}, \ldots, \beta_{n}^{\prime}$ as follows. For each $k \leq n$, we embed $\beta_{k}^{\prime}$ in $B_{k}$ so that the endpoints of $\beta_{k}$ are at $v_{k}$ and $w_{k}$ or both at $u_{k}$, and $\beta_{k}^{\prime}$ can be isotoped into $\Sigma_{k}$ fixing the endpoints of $\beta_{k}$. Now the only chains of length at least $m$ in $\Pi^{\prime}$ are contained in $\beta_{1}^{\prime}, \ldots, \beta_{n}^{\prime}$; and for each $j \leq r, \beta_{k}^{\prime}$ contains a chain of length $j m$ if and only if $\Gamma_{k}$ was in the orbit of $\Gamma_{j}$ under $\operatorname{TSG}_{+}(\Gamma)$. Now $\operatorname{TSG}_{+}\left(\Pi^{\prime}\right)$ is induced by a group of diffeomorphisms of $\left(S^{3}, \Pi^{\prime}\right)$ which leave $\left\{A_{1}, \ldots, A_{n}\right\}$ setwise invariant. Furthermore, there is a diffeomorphism of $\left(S^{3}, \Pi^{\prime}\right)$ which takes $A_{k}$ to itself interchanging its boundary components if and only if there is a diffeomorphism of $\left(S^{3}, \Gamma\right)$ which takes $A_{k}$ to itself interchanging its boundary components. Also if $g$ is a diffeomorphism of $\left(S^{3}, \Pi^{\prime}\right)$ which takes some $A_{k}$ to itself preserving its 
boundary components then $g$ induces a trivial automorphism on $\beta_{k}^{\prime}$. Now for each $\alpha \in \mathrm{TSG}_{+}(\Gamma)$, there exists $g_{\alpha} \in G$ such that $g_{\alpha}\left(\Pi^{\prime}\right)=\Pi^{\prime}$. If another element $g_{\alpha}^{\prime} \in G$ also induces $\alpha$ on $\Gamma$ and $g_{\alpha}^{\prime}\left(\Pi^{\prime}\right)=\Pi^{\prime}$, then $g_{\alpha}$ and $g_{\alpha}^{\prime}$ induce the same automorphism of $\Pi^{\prime}$. Define $\Phi: \mathrm{TSG}_{+}(\Gamma) \rightarrow \mathrm{TSG}_{+}\left(\Pi^{\prime}\right)$ by letting $\Phi(\alpha)$ denote the automorphism that $g_{\alpha}$ induces on $\Pi^{\prime}$. Then $N=\operatorname{ker}(\Phi)$. Now it is not hard to check that $\Phi$ is onto and hence $\mathrm{TSG}_{+}(\Pi) \cong H / N$.

Thus both $N$ and $H / N$ are realizable, and Claim 2 is proven.

Because of Claim 2, we can assume that if $g \in G$ such that $g\left(A_{i}\right)=A_{i}, g\left(c_{i}\right)=$ $c_{i}$, and $g\left(d_{i}\right)=d_{i}$, then $g$ induces a trivial automorphism on $\Gamma_{i}$. Thus we have completed Step 3.

Recall from Step 1 that the pared manifold $(W, W \cap(P \cup \Omega)$ ) is either Seifert fibered, $I$-fibered, or simple. We shall consider each of these cases in a separate step, making use of the above assumption.

Step 4. We prove the proposition when $(W, W \cap(P \cup \Omega))$ is Seifert fibered.

First we prove the following claim which does not assume that $(W, W \cap(P \cup \Omega))$ is Seifert fibered.

Claim 3. Let $T$ be the component of $\partial W$ which meets $\partial N(\Gamma)$. If $T$ is a torus, then $\mathrm{TSG}_{+}(\Gamma)$ is a subgroup of a dihedral group, and $\Gamma$ has a type II or type III sphere.

Proof of Claim 3. Let $\left\{x_{1}, \ldots, x_{r}\right\}$ denote those vertices of $\Gamma$ such that $\partial N\left(x_{i}\right)$ meets $W$. Let the components of $\partial N(V) \cap W$ be $J_{1}, \ldots, J_{q}$. (Note that for a given vertex $x_{i}$, the set $\partial N\left(x_{i}\right) \cap W$ may have more than one component, so we may have $q>r$.) Now each $J_{i}$ is a sphere with holes, and each boundary component of $J_{i}$ is either a boundary component of $\partial^{\prime} N(\varepsilon)$ for some edge $\varepsilon$, or a boundary component of some $A_{j}$. We saw in Step 1, that $\partial W-(W \cap(P \cup \Omega))$ is incompressible in $W$. Thus for each $i, J_{i}$ is incompressible in $W$, and hence each boundary component of $J_{i}$ is essential in $T$. Since $T$ is a torus, this means that every $J_{i}$ has exactly two boundary components.

Recall from Step 2 that $G$ permutes some of the $A_{j}$ 's or some components of $\partial N(V) \cap W$. Thus $W$ must contain at least two $A_{j}$ 's or at least two $J_{i}$ 's. In either case, $q>1$ and $T$ is made up of alternating annuli $R_{1}, \ldots, R_{q}$ (which are each either $A_{j}$ 's or components of $\left.\partial^{\prime} N(E)\right)$ and spheres with two holes, $J_{1}, \ldots, J_{q}$. Also, $G$ takes $T$ to itself, leaving each of the sets $\left\{J_{1}, \ldots, J_{q}\right\}$ and $\left\{R_{1}, \ldots, R_{q}\right\}$ setwise invariant. It follows that the group of automorphisms that $G$ induces on the set $\left\{J_{1}, \ldots, J_{q}, R_{1}, \ldots, R_{q}\right\}$ is a subgroup of the dihedral group $D_{q}$.

Define $\Phi: \mathrm{TSG}_{+}(\Gamma) \rightarrow D_{q}$ by letting $\Phi(a)$ denote the automorphism that $g_{a}$ induces on the set $\left\{J_{1}, \ldots, J_{q}, R_{1}, \ldots, R_{q}\right\}$. We see that $\Phi$ is well-defined as follows. Suppose that $g_{a}$ and $g_{a}^{\prime}$ are both elements of $G$ which induce $a$ on $\Gamma$. Then $g_{a}$ and $g_{a}^{\prime}$ induce the same permutation on the set of the components of $\partial N(V)$. Since $\Gamma$ 
has at most one edge between two vertices and every edge has two distinct vertices, $g_{a}$ and $g_{a}^{\prime}$ also induce the same permutation on the set of annuli $\left\{A_{1}, \ldots, A_{n}\right\}$, the set of circles $\left\{c_{1}, d_{1}, \ldots, c_{n}, d_{n}\right\}$, and the set of components of $\partial^{\prime} N(E)$. Thus $g_{a}$ and $g_{a}^{\prime}$ both induce the same permutation on the components of $\partial N(V) \cap W$ and on $\left\{J_{1}, \ldots, J_{q}, R_{1}, \ldots, R_{q}\right\}$. Therefore, $\Phi$ is a well-defined homomorphism.

We show as follows that $\Phi$ is one-to-one. Suppose that $\Phi(a)$ is the identity. Then for each $i=1, \ldots, q$, we have $g_{a}\left(J_{i}\right)=J_{i}$ and $g_{a}\left(R_{i}\right)=R_{i}$. Hence $a\left(x_{i}\right)=x_{i}$ for each vertex $x_{i}$ such that $\partial N\left(x_{i}\right)$ meets $W$. Let $v$ be a vertex of $\Gamma$ such that $v \notin\left\{x_{1}, \ldots, x_{r}\right\}$. Then for some $j$, the vertex $v$ is in $\Gamma_{j}$. Also since $T$ contains more than one $J_{i}$ and $g_{a}$ does not permute the $J_{i}$, the boundaries of $A_{j}$ cannot be interchanged by $g_{a}$. Thus by our assumption at the end of Step $3, a \mid \Gamma_{j}$ is the identity. In particular, $a(v)=v$. So $\Phi$ is one-to-one. Hence $\operatorname{TSG}_{+}(\Gamma)$ is a subgroup of $D_{q}$.

Finally, recall from Step 2 that if $\Gamma$ has no type II or III spheres, then $\partial N(V) \cap W$ contains a sphere with at least three holes. However, as we saw above, each $J_{i}$ is a sphere with two holes. Thus $\Gamma$ must have a type II or type III sphere. This completes the proof of Claim 3.

By Claim 3, if the component of $\partial W$ which meets $\partial N(\Gamma)$ is a torus then we are done. In particular, if $(W,(W \cap(P \cup \Omega)))$ is Seifert fibered, then we are done. Thus from now on, we assume that the component of $\partial W$ which meets $\partial N(\Gamma)$ is not a torus. Since $\partial W-(W \cap(P \cup \Omega))$ is incompressible in $W$, this component is also not a sphere. Hence this component has genus at least two. It follows that there is some vertex $v$ of $\Gamma$ such that $\partial N(v) \cap W$ contains a sphere with at least three holes.

Step 5. We prove the proposition when $(W, W \cap(P \cup \Omega))$ is I-fibered.

Since $W$ is an $I$-fibered subspace of $S^{3}, W=Y \times I$ where $Y$ is a surface with holes and the $I$-fibers come from the $I$-factor in the product. Now, since $(W, W \cap(P \cup \Omega)$ ) is $I$-fibered as a pared manifold, $W \cap(P \cup \Omega)=\partial Y \times I$. Thus $Y_{0}=Y \times\{0\}$ and $Y_{1}=Y \times\{1\}$ are components of $\partial N(V) \cap W$. In particular there are vertices $v_{0}$ and $v_{1}$ in $\Gamma$ such that either $v_{0} \neq v_{1}$, and $Y_{0}=\partial N\left(v_{0}\right) \cap W$ and $Y_{1}=\partial N\left(v_{1}\right) \cap W$, or $v_{0}=v_{1}$ and both $Y_{0}$ and $Y_{1}$ are components of $\partial N\left(v_{0}\right) \cap W$. In either case, $Y_{0}$ and $Y_{1}$ are spheres with $r$ holes, for some $r$. By our assumption at the end of Step 4, $\partial W$ has genus at least two. Thus $r \geq 3$. Let $b_{1}, \ldots, b_{r}$ denote the boundary components of $Y$, and for each $i$, let $C_{i}=b_{i} \times I$. Since $W \cap(P \cup \Omega)=\partial Y \times I$, each $C_{i}$ is either an element of $\Omega$ or a component of $\partial^{\prime} N(E)$. For each $i$, let $F_{i}$ denote the sphere or pinched sphere obtained from $C_{i}$ by adding disks within $N\left(v_{0}\right)$ and $N\left(v_{1}\right)$ which are disjoint from $\Gamma$ and from each other except at $v_{0}$ and $v_{1}$. Let $E_{i}$ denote the closure of the component of $S^{3}-F_{i}$ which is disjoint from $W$, and let $\gamma_{i}=\Gamma \cap E_{i}$. Now $\Gamma=\gamma_{1} \cup \cdots \cup \gamma_{r}$. If $C_{i} \in \Omega$ then $\gamma_{i}=\Gamma_{j}$ for some $j$, and if $C_{i}$ is a component of $\partial^{\prime} N(E)$ then $\gamma_{i}$ is a single edge. Since $\partial X-P$ is incompressible in $X, Y_{0}$ is incompressible in $X$. Thus no $b_{i} \times\{0\}$ bounds a disk in $X$. Since $W$ has only one 
boundary component, $X=\operatorname{cl}\left(S^{3}-N(\Gamma)\right)$. It follows that no $b_{i} \times\{0\}$ bounds a disk in $E_{i}$ disjoint from $\Gamma$, and hence at most one $\gamma_{i}$ is a single vertex. So if $v_{0}=v_{1}$, then $\Gamma$ has a type III sphere.

Suppose that $\Gamma$ has no type II or III spheres. Thus $v_{0} \neq v_{1}$ and by Step 2 every $\gamma_{i}$ is an arc. For the sake of contradiction suppose that $\partial Y$ has at least four components. Let $c$ be a simple closed curve on $Y \times\{0\}$ which separates two of the boundary components of $Y$ from the other boundary components of $Y$. Let $A$ denote the annulus $c \times I$ in $Y \times I$. Now $c \times\{0\}$ bounds a disk $D_{0}$ in $N\left(v_{0}\right)$ such that $D_{0} \cap \Gamma=\left\{v_{0}\right\}$ and $c \times\{1\}$ bounds a disk $D_{1}$ in $N\left(v_{1}\right)$ such that $D_{1} \cap \Gamma=\left\{v_{1}\right\}$. Let $\Sigma=A \cup D_{0} \cup D_{1}$. Then $\Sigma$ is a type II sphere, contrary to our hypothesis. Since we already know that $r \geq 3$, it follows that $r=3$. Hence the underlying abstract graph of $\Gamma$ is a graph, $\theta_{3}$, containing two vertices of valence three and some positive number vertices of valence 2 . Now $\operatorname{Aut}\left(\theta_{3}\right)$ is a subgroup of $S_{3} \times \mathbb{Z}$. Thus $\mathrm{TSG}_{+}(\Gamma)$ is a subgroup of the dihedral group $D_{6} \cong S_{3} \times \mathbb{Z}_{2}$ and hence is isomorphic to a finite subgroup of Diff $+\left(S^{3}\right)$. Furthermore, we can embed $\theta_{3}$ in $S^{2}$ as $\Delta$ such that the vertices of valence three are at the poles of the sphere, and the components of $S^{2}-\Delta$ are three identical wedges. Thus $H=\operatorname{TSG}_{+}(\Gamma) \leq \operatorname{Aut}\left(\theta_{3}\right)=\operatorname{TSG}_{+}(\Delta)$, and $\mathrm{TSG}_{+}(\Delta)$ is induced by an isomorphic finite subgroup of $\mathrm{SO}(4)$. Thus if $\Gamma$ has no type II or III spheres, then we are done.

Now we return to the general case. Recall that every $g \in G$ restricts to a map of the pair $(W, W \cap(P \cup \Omega))$. So for every $g \in G, g\left(\left\{Y_{0}, Y_{1}\right\}\right)=\left\{Y_{0}, Y_{1}\right\}$ and $g\left(\left\{C_{1}, \ldots, C_{r}\right\}\right)=\left\{C_{1}, \ldots, C_{r}\right\}$. Suppose that there is some $g \in G$ which interchanges $Y_{0}$ and $Y_{1}$. Let $\Phi: \mathrm{TSG}_{+}(\Gamma) \rightarrow \mathbb{Z}_{2}$ be defined as follows. For each $a \in \mathrm{TSG}_{+}(\Gamma)$, let $\Phi(a)$ be the permutation that $g_{a}$ induces on the set $\left\{Y_{0}, Y_{1}\right\}$. Then $\Phi$ is onto. If $\operatorname{ker}(\Phi)$ is trivial then $\mathrm{TSG}_{+}(\Gamma) \cong \mathbb{Z}_{2}$. Hence in this case we are done. If $N=\operatorname{ker}(\Phi)$ is non-trivial, then we let $\Pi$ be the embedded graph obtained from $\Gamma$ by adding a single edge incident to $v_{0}$. Then $\mathrm{TSG}_{+}(\Pi) \cong \operatorname{ker}(\Phi)$. Hence both $N$ and $H / N \cong \mathbb{Z}_{2}$ are realizable. Thus from now on we assume that for every $g \in G$ we have $g\left(Y_{0}\right)=Y_{0}$ and $g\left(Y_{1}\right)=Y_{1}$.

Now suppose that there is some $a \in \mathrm{TSG}_{+}(\Gamma)$ such that $a\left(\gamma_{j}\right)=\gamma_{j}$ for some $j$. If $C_{j} \in \Omega$, then $C_{j}=A_{i}$, for some $A_{i}$. Hence $\gamma_{j}=\Gamma_{i}, g_{a}\left(A_{i}\right)=A_{i}$, and since $g_{a}\left(Y_{0}\right)=Y_{0}$ and $g_{a}\left(Y_{1}\right)=Y_{1}, g_{a}\left(c_{i}\right)=c_{i}$ and $g_{a}\left(d_{i}\right)=d_{i}$. Thus by our assumption at the end of Step 3, $a$ induces the trivial automorphism on $\gamma_{j}$. If $C_{j} \notin \Omega$, then $\gamma_{j}$ is a single edge. Thus again, since $g_{a}\left(Y_{0}\right)=Y_{0}$ and $g_{a}\left(Y_{1}\right)=Y_{1}, a$ induces the trivial automorphism on $\gamma_{j}$. Thus for every non-trivial $a \in \mathrm{TSG}_{+}(\Gamma)$, there is some $\gamma_{j}$ such that $a\left(\gamma_{j}\right) \neq \gamma_{j}$. Because $\mathrm{TSG}_{+}(\Gamma)$ is non-trivial, without loss of generality we can assume that $\gamma_{1}$ is not setwise invariant under $\operatorname{TSG}_{+}(\Gamma)$. Let $\left\{\gamma_{1}, \ldots, \gamma_{q}\right\}$ be the orbit of $\gamma_{1}$ under $\operatorname{TSG}_{+}(\Gamma)$. Now define $\Phi: \operatorname{TSG}_{+}(\Gamma) \rightarrow S_{q}$ by letting $\Phi(a)$ be the permutation that $a$ induces on the set $\left\{\gamma_{1}, \ldots, \gamma_{q}\right\}$. Then $\Phi$ is a homomorphism.

We prove that $\Phi$ is onto as follows. Let $(i j)$ be a transposition in $S_{q}$. Since $\left\{\gamma_{1}, \ldots, \gamma_{q}\right\}$ is the orbit of $\gamma_{1}$, there is some $a \in \operatorname{TSG}_{+}(\Gamma)$, such that $a\left(\gamma_{i}\right)=\gamma_{j}$. 
We will define an element $g \in G$ as follows. Let $g\left|E_{i}=g_{a}\right| E_{i}$ and $g\left|E_{j}=g_{a}^{-1}\right| E_{j}$. Then $g$ interchanges $E_{i}$ and $E_{j}$. Let $b$ denote a simple closed curve in $Y$ which separates $b_{i}$ and $b_{j}$ from all the other boundary components of $Y$. Let $F$ denote the disk with two holes in $Y$ bounded by the three curves $b_{i}, b_{j}$, and $b$. Extend $g$ to a diffeomorphism of $F \times I$ such that $g \mid(b \times I)$ is the identity. Next extend $g$ to $S^{3}-\left((F \times I) \cup N\left(v_{0}\right) \cup N\left(v_{1}\right)\right)$ by the identity. Finally, extend $g$ within $N\left(v_{0}\right)$ and $N\left(v_{1}\right)$ such that $g(\Gamma)=\Gamma$. Now $g:\left(S^{3}, \Gamma\right) \rightarrow\left(S^{3}, \Gamma\right)$ and $g \mid \Gamma-\left(\gamma_{i} \cup \gamma_{j}\right)$ is the identity. Let $a^{\prime}$ denote the automorphism of $\Gamma$ induced by $g$. Then $a^{\prime}$ interchanges $\gamma_{i}$ and $\gamma_{j}$, and $a^{\prime} \mid \Gamma-\left(\gamma_{i} \cup \gamma_{j}\right)$ is the identity. So $\Phi(a)=(i j)$, and hence $\Phi$ is onto. If $\operatorname{ker}(\Phi)$ is trivial then $\mathrm{TSG}_{+}(\Gamma) \cong S_{q}$ and we are done.

If $N=\operatorname{ker}(\Phi)$ is non-trivial, then $r-q \geq 2$. If $v_{0} \neq v_{1}$, we let $\Pi$ be the embedded graph obtained from $\gamma_{q+1} \cup \cdots \cup \gamma_{r}$ by adding a single edge at $v_{0}$ and adding a chain of length $m$ at $v_{1}$. If $v_{0}=v_{1}$, then each $\gamma_{i}$ contains at least two vertices in its interior. In this case, let $e$ be an edge in $\gamma_{q+1}$ such that $e$ contains $v_{0}$. Now we add a vertex to the interior of every edge in the orbit of $e$ under $N$, and add a single edge at each of these new vertices. Let $\Pi$ be $\gamma_{q+1} \cup \cdots \cup \gamma_{r}$ together with these new vertices and edges. Now in either case, $\operatorname{TSG}_{+}(\Pi) \cong N$. Also let $\Pi^{\prime}$ denote the graph obtained by gluing $q$ edges together at a single vertex to form a star. Then $\mathrm{TSG}_{+}\left(\Pi^{\prime}\right) \cong S_{q}=H / N$. Hence both $N$ and $H / N$ are realizable. Thus we are done in the case where $(W, W \cap(P \cup \Omega))$ is $I$-fibered.

Step 6. We prove the proposition when $(W, W \cap(P \cup \Omega))$ is simple.

Note that the argument in the beginning of this step will be similar to the analogous part of the proof of Theorem 1 in [F2].

We assume by Steps 4 and 5 that $(W, W \cap(P \cup \Omega))$ is neither Seifert fibered nor $I$ fibered. Now by applying Thurston's Hyperbolization Theorem for Pared Manifolds [Th] to $(W, W \cap(P \cup \Omega))$ we conclude that $W-(W \cap(P \cup \Omega))$ admits a finite volume complete hyperbolic metric with totally geodesic boundary. Let $D$ denote the double of $W-(W \cap(P \cup \Omega))$ along its boundary. Then $D$ is a finite volume hyperbolic manifold. For every $a \in \mathrm{TSG}_{+}(\Gamma)$, the diffeomorphism $g_{a} \mid W$ defines a diffeomorphism $g_{a}^{\prime}$ of $D$ which restricts to $g_{a}$ on each side of $D$. Now we use Mostow's Rigidity Theorem [Mo], to obtain an orientation preserving finite order isometry $f_{a}^{\prime}$ of $D$ that restricts to an isometry $f_{a}$ of $W-(W \cap(P \cup \Omega))$ such that $f_{a}$ is homotopic to $g_{a} \mid W-(W \cap(P \cup \Omega))$. Furthermore, Mostow's Rigidity Theorem implies that the set of all such $f_{a}$ generates a finite group $K^{\prime}$ of isometries of $W-(W \cap(P \cup \Omega))$. Now by removing horocyclic neighborhoods of the cusps of $W-(W \cap(P \cup \Omega))$, we obtain a copy of the pair $(W, W \cap(P \cup \Omega))$ which is contained in $W-(W \cap(P \cup \Omega))$ and is setwise invariant under $K^{\prime}$. We shall abuse notation slightly and consider $K^{\prime}$ to be a finite group of isometries of $(W, W \cap(P \cup \Omega)$ ) rather than of this copy. Now $K^{\prime}$ restricts to a finite group of isometries of the tori and annuli in $W \cap(P \cup \Omega)$ with respect to a flat metric. Finally, it follows from 
Waldhausen's Isotopy Theorem [Wa] that each $f_{a}$ is isotopic to $g_{a} \mid W$ by an isotopy leaving $W \cap(P \cup \Omega)$ setwise invariant.

Now observe that since $\partial N(V) \cap W, \partial^{\prime} N(E) \cap W,\left\{A_{1}, \ldots, A_{n}\right\}$, and $\Theta \cap W$ are each setwise invariant under $G$, each of these sets is also setwise invariant under the isometry group $K^{\prime}$. Let $f$ and $g$ be elements of $K^{\prime}$ which induce the same permutation of the components of $\partial N(V) \cap W, \partial^{\prime} N(E) \cap W$, and $\left\{A_{1}, \ldots, A_{n}\right\}$. We prove as follows that $f=g$. By the end of Step 4 we know there is some vertex $v$ of $\Gamma$ such that a component $J$ of $\partial N(v) \cap W$ is a sphere with $r \geqslant 3$ holes. Let $\alpha_{1}, \ldots, \alpha_{r}$ denote the boundary components of $J$. Now $f(J)=g(J)$ and $f\left(\alpha_{i}\right)=g\left(\alpha_{i}\right)$ for each $i=1, \ldots, r$. Hence $f^{-1} \circ g$ restricts to a finite order orientation preserving diffeomorphism of $J$ which setwise fixes all of the boundary components of $J$. Since $J$ is a sphere with at least three holes, this implies that $f^{-1} \circ g \mid J$ is the identity. Finally, since $f$ and $g$ are isometries of $W$ which are identical on $J$, we must have $f=g$.

Now suppose that $f_{a}$ and $f_{a}^{\prime}$ are elements of $K^{\prime}$ which come from elements $g_{a}$ and $g_{a}^{\prime}$ of $G$ respectively, such that $g_{a}$ and $g_{a}^{\prime}$ both induce a given $a$ on $\Gamma$. As in the proof of Step 4, $g_{a}$ and $g_{a}^{\prime}$ induce the same permutation of the components of $\partial N(V) \cap W$, $\partial^{\prime} N(E) \cap W$, and $\left\{A_{1}, \ldots, A_{n}\right\}$. Hence $f_{a}$ and $f_{a}^{\prime}$ also induce the same permutation of these components. So by the above paragraph $f_{a}=f_{a}^{\prime}$. Thus each $a \in \mathrm{TSG}_{+}(\Gamma)$ determines a unique $f_{a} \in K^{\prime}$. Also if $a, b \in \mathrm{TSG}_{+}(\Gamma)$, then $g_{a} \circ g_{b}$ and $g_{a \circ b}$ induce the same permutation of the components of $\partial N(V) \cap W, \partial^{\prime} N(E) \cap W$, and $\left\{A_{1}, \ldots, A_{n}\right\}$. Thus $f_{a} \circ f_{b}$ and $f_{a \circ b}$ induce the same permutation of each of these sets. Hence by the above paragraph $f_{a} \circ f_{b}=f_{a \circ b}$. Thus $K^{\prime}=\left\{f_{a} \mid a \in \mathrm{TSG}_{+}(\Gamma)\right\}$.

Now we will re-embed $W$ in $S^{3}$ and extend $K^{\prime}$ to a finite subgroup of $\operatorname{Diff}_{+}\left(S^{3}\right)$. We start with $W^{\prime}=W \cup N\left(\Gamma^{\prime}\right) \cup\left(B_{1} \cup \cdots \cup B_{n}\right)$. Then $\partial W^{\prime}$ is a collection of tori in $\Theta$. We re-embed $W^{\prime}$ in $S^{3}$ as follows. Let $T_{j}$ be a component of $\partial W^{\prime}$. Because $T_{j}$ is incompressible in $M$, the closure of the component of $S^{3}-T_{j}$ contained in $M$ is a knot complement $R_{j}$. Now, up to isotopy, there is a well-defined longitude $\ell_{j}$ of $T_{j}$, which bounds a Seifert surface in $R_{j}$. Also for every $g \in G$, there is a component $T_{k}$ of $\partial W^{\prime}$ such that $g\left(T_{j}\right)=T_{k}$. Now $T_{k}$ bounds a knot complement $R_{k}$ in $M, g\left(R_{j}\right)=R_{k}$, and $g\left(\ell_{j}\right)$ is a longitude of $R_{k}$. We re-embed $W^{\prime}$ in $S^{3}$ by replacing each knot complement $R_{j}$ by a solid torus $U_{j}$ such that a meridian of $U_{j}$ is glued to $\ell_{j}$. Re-embedding $W^{\prime}$ in $S^{3}$ in this way gives us a re-embedding $\Delta^{\prime}$ of $\Gamma^{\prime}$.

We will extend $K^{\prime}$ to a finite group of diffeomorphisms of $S^{3}$ in stages. For each ball or pinched ball $B_{i}$, let $C_{i}$ denote the solid cylinder obtained from $B_{i}$ by removing $\operatorname{int}\left(N\left(v_{i}\right) \cup N\left(w_{i}\right)\right)$ or $\operatorname{int}\left(N\left(u_{i}\right)\right)$. After we have re-embedded $W^{\prime}$ in $S^{3}$ as described above, $S^{3}-W$ consists of the solid tori $U_{1}, \ldots, U_{q}$, the balls $N(v)$ where $v \in \Delta^{\prime}$, and the solid cylinders $C_{1}, \ldots, C_{n}$ and $N(e)$ where $e \subset \Delta^{\prime}$. First we will extend $K^{\prime}$ to the solid tori $U_{1}, \ldots, U_{q}$. Recall that each $f_{a} \in K^{\prime}$ is isotopic to the corresponding $g_{a}$ on $(W, W \cap(P \cup \Omega))$ and each $g_{a}$ took longitudes to longitudes on $\partial R_{1} \cup \cdots \cup \partial R_{q}$. Thus $K^{\prime}$ will take meridians to meridians on $\partial U_{1} \cup \cdots \cup \partial U_{q}$. 
Since $K^{\prime} \mid\left(\partial U_{1} \cup \cdots \cup \partial U_{q}\right)$ is a finite group of orientation preserving isometries, we can find a finite number of pairwise disjoint meridians for each $U_{i}$ such that the collection $\left\{\mu_{1}, \ldots, \mu_{r}\right\}$ of meridians for $\partial U_{1} \cup \cdots \cup \partial U_{q}$ are setwise invariant under $K^{\prime}$. We first extend $K^{\prime}$ radially from $\left\{\mu_{1}, \ldots, \mu_{r}\right\}$ to a collection of pairwise disjoint meridional disks bounded by $\left\{\mu_{1}, \ldots, \mu_{r}\right\}$. These disks cut the solid tori $U_{1}, \ldots, U_{q}$ into a collection of solid cylinders. We can now extend $K^{\prime}$ radially within these solid cylinders to obtain a finite group $K_{1}$ of diffeomorphisms of $W_{1}=W \cup U_{1} \cup \cdots \cup U_{q}$.

Next we will extend $K_{1}$ to the balls $N(v)$ such that $v \in \Delta^{\prime}$. Observe that $K_{1}$ restricts to a finite group of diffeomorphisms of the spheres with holes $\bigcup_{v \in \Delta^{\prime}} \partial N(v) \cap$ $W$. We extend $K_{1}$ radially first to the collection of spheres $\bigcup_{v \in \Delta^{\prime}} \partial N(v)$ and then to the balls $\bigcup_{v \in \Delta^{\prime}} N(v)$ to obtain a finite group $K_{2}$ of diffeomorphisms of $W_{1} \cup$ $\bigcup_{v \in \Delta^{\prime}} N(v)$ which leaves $\bigcup_{v \in \Delta^{\prime}} N(v) \cap \Delta^{\prime}$ setwise invariant.

Now $K_{2}$ restricts to finite groups of diffeomorphisms of the annuli $A_{1}, \ldots, A_{n}$, of the disks $B_{i} \cap\left(\partial N\left(v_{i}\right) \cup \partial N\left(w_{i}\right)\right)$ or $B_{i} \cap \partial\left(N\left(u_{i}\right)\right)$ for $i=1, \ldots, n$, of the annuli $\partial^{\prime} N(e)$ for each $e \subset \Delta^{\prime}$, and of the disks $\partial N(e) \cap N(V)$ for each $e \subset \Delta$. Thus we can extend $K_{2}$ radially within each of the solid cylinders $C_{1}, \ldots, C_{n}$ and each $N(e)$ where $e \subset \Delta^{\prime}$, to obtain a finite group of diffeomorphisms $K$ of $S^{3}$ such that the collection of the cores of these solid cylinders is setwise invariant. In particular, $E \cap \Delta^{\prime}$ is setwise invariant. Thus $K$ is a finite subgroup of $\operatorname{Diff}_{+}\left(S^{3}\right)$ which leaves $\Delta^{\prime}$ setwise invariant.

Now for each $f_{a} \in K^{\prime}$, let $h_{a}$ denote the element of $K$ obtained from $f_{a}$ by the above extensions. Since $f_{a}$ is isotopic to $g_{a}$ on $(W, W \cap(P \cup \Omega)), h_{a}$ is isotopic to $g_{a}$ on $(W, W \cap(P \cup \Omega))$. Hence $h_{a}$ induces $a \mid \Delta^{\prime}$. Define $\Phi: H \rightarrow K$ by $\Phi(a)=h_{a}$. Then $\Phi$ is well defined since each $a \in \mathrm{TSG}_{+}(\Gamma)$ determines a unique $f_{a} \in K^{\prime}$, which in turn extends to a unique $h_{a} \in K$. Also, every $g \in K$ came from such an $a \in \mathrm{TSG}_{+}(\Gamma)$. Thus $\Phi$ is onto. To see that $\Phi$ is one-to-one, suppose that $a \in \mathrm{TSG}_{+}(\Gamma)$ such that $\Phi(a)=h_{a}$ is the identity on $S^{3}$. Thus $h_{a} \mid W$ is the identity. So for every vertex $v$ of $\Gamma^{\prime}, a(v)=v$. Let $v$ be a vertex of $\Gamma$ such that $\partial N(v)$ does not meet $W$. Then $v$ is a vertex of some $\Gamma_{i}$. Now $A_{i}$ is contained in $\partial W$, and $h_{a} \mid A_{i}$ is the identity. Thus $g_{a}\left(A_{i}\right)=A_{i}, g_{a}\left(c_{i}\right)=c_{i}$, and $g_{a}\left(d_{i}\right)=d_{i}$. Now by our assumption at the end of Step $3, a \mid \Gamma_{i}$ is the identity. So $a(v)=v$. It follows that $\Phi$ is one-to-one, and hence $H \cong K$.

Finally, suppose that $\Gamma$ has no type II or type III spheres. As we saw in Step 2, then each $\Gamma_{i}$ is either an arc or a single vertex. Let $\Delta$ denote the graph obtained from $\Delta^{\prime}$ by adding the core of each $C_{i}$ together with additional vertices so that $\Delta$ is a re-embedding of $\Gamma$ that is setwise invariant under $K$. Now for each $a \in \mathrm{TSG}_{+}(\Gamma)$, $h_{a}$ induces $a$ on $\Delta$. It follows that $H$ is a subgroup of $\operatorname{TSG}_{+}(\Delta)$. Thus $H$ is induced on $\Delta$ by an isomorphic finite subgroup of $\operatorname{Diff}_{+}\left(S^{3}\right)$. This completes the proof of Proposition 1. 


\section{Proof of Proposition 2}

An essential part of the proof of Proposition 2 involves showing that if $\operatorname{TSG}_{+}(\Gamma)$ is a simple group, then we can find an embedded graph $\Delta$ with no type I vertices such that $\mathrm{TSG}_{+}(\Delta) \cong \mathrm{TSG}_{+}(\Gamma)$. Our strategy is to first find an invariant proper subgraph $\Gamma^{\prime}$ of $\Gamma$, then create a new graph $\Lambda$ containing $\Gamma^{\prime}$ such that $\operatorname{TSG}_{+}(\Lambda) \cong \operatorname{TSG}_{+}(\Gamma)$ and $\Lambda$ has fewer type I vertices than $\Gamma$. Continuing this process until no type I vertices remain, we arrive at the desired embedded graph $\Delta$.

We begin with some definitions and lemmas.

Definition 7. Let $\Gamma$ be a graph embedded in $S^{3}$, let $\Sigma$ be a type I sphere for $\Gamma$, let $B$ be the closure of one of the components of $S^{3}-\Sigma$, let $\alpha=B \cap \Gamma$, and let $\{v\}=\Sigma \cap \Gamma$. Then we say that $\alpha$ is a type I subgraph of $\Gamma$ with associated sphere $\Sigma$, and associated type I ball $B$, and $v$ is the attaching vertex of $\alpha, \Sigma$ or $B$. If $X$ and $X^{\prime}$ are subsets of $S^{3}$ such that $X \cap X^{\prime}$ is either empty or a single vertex of $\Gamma$, then we say that $X$ and $X^{\prime}$ are almost disjoint.

Lemma 1. Let $\Gamma$ be an embedded graph, and let $\Gamma_{1}, \ldots, \Gamma_{r}$ be pairwise almost disjoint type I subgraphs with attaching vertices $v_{1}, \ldots, v_{r}$, respectively. Let $\Gamma^{\prime}=$ $\operatorname{cl}\left(\Gamma-\left(\Gamma_{1} \cup \cdots \cup \Gamma_{r}\right)\right)$ and let $\alpha$ be a type I subgraph of $\Gamma^{\prime}$ with attaching vertex $x$. Then $\Gamma$ has a type I ball $E$ with attaching vertex $x$, and pairwise almost disjoint type $I$ balls $E_{1}, \ldots, E_{r}$ associated with $\Gamma_{1}, \ldots, \Gamma_{r}$ such that $E \cap \Gamma^{\prime}=\alpha$ and $E$ is almost disjoint from $E_{i}$ for all $i$ such that $v_{i} \in \operatorname{cl}\left(\Gamma^{\prime}-\alpha\right)$.

Proof. Let $D$ be a type I ball for $\Gamma^{\prime}$ associated with $\alpha$. For each $i$, let $B_{i}$ and $C_{i}$ be type I balls for $\Gamma$ associated with $\Gamma_{i}$ such that $B_{i}-\left\{v_{i}\right\} \subset \operatorname{int}\left(C_{i}\right)$. Then for each $i \neq j, \Gamma_{i}$ is almost disjoint from $C_{j}$. Now for each $i$, we can find a small ball $B_{i}^{\prime} \subset B_{i}$ such that $v_{i} \in B_{i}^{\prime}$ and $B_{i}^{\prime}$ is almost disjoint from each $C_{j}$ with $j \neq i$ and from $\partial D$.

We start by shrinking $B_{1}$ within itself to $B_{1}^{\prime}$ by an isotopy of $S^{3}$ which is pointwise fixed on $\operatorname{cl}\left(S^{3}-C_{1}\right)$. This isotopy takes $\Gamma_{1}$ to some $\Gamma_{1}^{\prime} \subset B_{1}^{\prime}$. Next we shrink $B_{2}$ to $B_{2}^{\prime}$ fixing $\operatorname{cl}\left(S^{3}-C_{2}\right)$, taking $\Gamma_{2}$ to some $\Gamma_{2}^{\prime} \subset B_{2}^{\prime}$. Continue this process for $i=1, \ldots, r$. The composition of these isotopies is an isotopy of $S^{3}$ which is fixed on $\Gamma^{\prime}$ and takes $\Gamma$ to $\Lambda=\Gamma^{\prime} \cup \Gamma_{1}^{\prime} \cup \cdots \cup \Gamma_{r}^{\prime}$. Now $B_{1}^{\prime}, \ldots, B_{r}^{\prime}$ are pairwise almost disjoint type I balls for $\Lambda$ with associated subgraphs $\Gamma_{1}^{\prime}, \ldots, \Gamma_{r}^{\prime}$ such that each $B_{i}^{\prime}$ is almost disjoint from $\partial D$.

Now without loss of generality we can choose $s$ and $t$ such that if $i \leq s$ then $B_{i}^{\prime} \subset \operatorname{int}(D)$, if $s<i \leq t$ then $B_{i}^{\prime} \subset \operatorname{cl}\left(S^{3}-D\right)$, and if $t<i \leq r$ then $v_{i}=x$ and $B_{i}^{\prime} \subset D$. Let $B \subset D$ be a ball containing $B_{t+1}^{\prime} \cup \cdots \cup B_{r}^{\prime}$ which is almost disjoint from $\Gamma^{\prime}$ and from each $B_{i}^{\prime}$ with $i \leq s$. Let $B^{\prime} \subset \operatorname{cl}\left(S^{3}-D\right)$ be a ball with $x \in \partial B^{\prime}$ which is almost disjoint from $\Gamma^{\prime}$ and from each $B_{i}^{\prime}$ with $s<i \leq t$. Now there is an isotopy of $S^{3}$ taking $B$ to $B^{\prime}$ and pointwise fixing $\Gamma^{\prime} \cup\left(B_{1}^{\prime} \cup \cdots \cup B_{t}^{\prime}\right)$. For each $i$, 
let $B_{i}^{\prime \prime}$ and $\Gamma_{i}^{\prime \prime}$ denote the images of $B_{i}^{\prime}$ and $\Gamma_{i}^{\prime}$ as a result of this isotopy. This isotopy takes $\Lambda$ to $\Lambda^{\prime}=\Gamma^{\prime} \cup \Gamma_{1}^{\prime \prime} \cup \cdots \cup \Gamma_{r}^{\prime \prime}$.

Now $B_{1}^{\prime \prime}, \ldots, B_{r}^{\prime \prime}$ are pairwise almost disjoint type I balls for $\Lambda^{\prime}$ with associated subgraphs $\Gamma_{1}^{\prime \prime}, \ldots, \Gamma_{r}^{\prime \prime}$ respectively. Also $D$ is a type I ball for $\Lambda^{\prime}$ such that $D \cap \Gamma^{\prime}=\alpha$, and $D$ is almost disjoint from $B_{i}^{\prime \prime}$ for all $i$ such that $v_{i} \in \operatorname{cl}\left(\Gamma^{\prime}-\alpha\right)$. The composition of the above isotopies determines a homeomorphism $h:\left(S^{3}, \Gamma\right) \rightarrow\left(S^{3}, \Lambda^{\prime}\right)$ which is fixed on $\Gamma^{\prime}$ and takes each $\Gamma_{i}$ to $\Gamma_{i}^{\prime \prime}$. Now let $E=h^{-1}(D)$, and for each $i$, let $E_{i}=h^{-1}\left(B_{i}^{\prime \prime}\right)$. Then the balls $E$ and $E_{1}, \ldots, E_{r}$ satisfy the conclusion of the lemma.

We would like to focus on those type I subgraphs which are as small as possible.

Definition 8. Let $\Gamma$ be a graph embedded in $S^{3}$ and let $\alpha$ be a type I subgraph with associated sphere $\Sigma$, associated ball $B$, and attaching vertex $v$. Suppose that $\Gamma$ has no type I subgraph which is a proper subset of $\alpha$. Then we say that $\alpha$ is an innermost subgraph and $B$ is an innermost ball of $\Gamma$.

We can also choose a particular type I vertex, and look for the smallest type I subgraph attached at that vertex.

Definition 9. Let $\Gamma$ be a graph embedded in $S^{3}$ and let $\alpha$ be a type I subgraph with associated sphere $\Sigma$, associated ball $B$, and attaching vertex $v$. Suppose that $\Gamma$ has no type I subgraph with attaching vertex $v$ which is a proper subset of $\alpha$. Then we say that $\alpha$ is an innermost subgraph rel $v$ and $B$ is an innermost ball rel $v$.

Observe that if $\alpha$ is any type I subgraph of $\Gamma$ with attaching vertex $v$, then $\alpha$ contains an innermost subgraph and an innermost subgraph rel $v$. The following lemma can be proved using an argument identical to that of Lemma 4.1 of [Su], so we do not include the proof here.

Lemma 2. Let $\Gamma$ be an embedded graph and let $\lambda$ be an innermost subgraph of $\Gamma$ (respectively rel $v$ ). Let $\Sigma$ be a type I sphere for $\Gamma$ (respectively rel $v$ ) and let the closures of the components of $\Gamma-\Sigma$ be $\alpha$ and $\beta$. Then $\lambda$ is contained entirely in either $\alpha$ or $\beta$.

Observe that it follows from Lemma 2 that if $\lambda_{1}$ and $\lambda_{2}$ are innermost subgraphs of $\Gamma$ (respectively rel $v$ ) which are not almost disjoint, then $\lambda_{1}=\lambda_{2}$. Thus every embedded graph $\Gamma$ has a unique collection of innermost subgraphs (respectively rel $v$ ) and these subgraphs are pairwise almost disjoint. Hence any diffeomorphism of $\left(S^{3}, \Gamma\right)$ takes this unique collection to itself.

Lemma 3. Let $\Gamma$ be an embedded graph with a type I vertex. Then $\Gamma$ has a type I vertex $v$ with the property that at most one innermost subgraph rel $v$ is not an innermost subgraph of $\Gamma$. 
Proof. Suppose that $v_{1}$ is a type I vertex of $\Gamma$ such that some innermost subgraph rel $v_{1}$ is not an innermost subgraph of $\Gamma$. Call this subgraph $\Gamma_{1}$. Since $\Gamma_{1}$ is not innermost, it contains a vertex $v_{2} \neq v_{1}$ where an innermost subgraph is attached. Consider those innermost subgraphs of $\Gamma$ rel $v_{2}$ which do not contain $v_{1}$. Suppose that one of these subgraphs is not innermost. Call this subgraph $\Gamma_{2}$. Since $\Gamma_{2}$ does not contain $v_{1}, \Gamma_{2}$ is a proper subgraph of $\Gamma_{1}$.

Repeat the above argument starting with $\Gamma_{2}$ in place of $\Gamma_{1}$. Since the graph $\Gamma$ is finite, it cannot have an infinite nested sequence of proper subgraphs. Hence eventually, we obtain a vertex $v_{n}$ such that every innermost subgraph of $\Gamma$ rel $v_{n}$ that does not contain $v_{n-1}$ is innermost. Since $\Gamma$ has precisely one innermost subgraph rel $v_{n}$ that contains $v_{n-1}$, the vertex $v_{n}$ satisfies the conclusion of the lemma.

Now we are ready to prove Proposition 2.

Proposition 2. Let $\Gamma$ be an embedded graph, and let $H=\mathrm{TSG}_{+}(\Gamma)$. Then either $H$ is realizable by a graph with no type I spheres, $H \cong S_{r}$ for some $r$, or $H$ has a non-trivial normal subgroup $N$ such that both $N$ and $H / N$ are realizable.

Proof. If $\mathrm{TSG}_{+}(\Gamma) \cong \mathbb{Z}_{2}$, then $H$ is realized by the graph consisting of a single edge, and if $H$ is trivial then it is realized by the graph consisting of a single vertex. Thus we assume that $H \neq \mathbb{Z}_{2}, H$ is not trivial, and $\Gamma$ has at least one type I sphere. We will show that either $H \cong S_{r}$ for some $r, H$ has a non-trivial normal subgroup $N$ such that both $N$ and $H / N$ are realizable, or there exists an embedded graph $\Lambda$ such that $\mathrm{TSG}_{+}(\Lambda) \cong \mathrm{TSG}_{+}(\Gamma)$ and $\Lambda$ has fewer type I vertices than $\Gamma$.

By Lemma 3 we can choose an innermost type I vertex $v_{1}$ which has the property that at most one innermost subgraph rel $v_{1}$ is not an innermost subgraph of $\Gamma$. If $v_{1}$ is fixed by $\mathrm{TSG}_{+}(\Gamma)$, let $\left\{\Gamma_{1}, \ldots, \Gamma_{n}\right\}$ be the collection of innermost subgraphs of $\Gamma$ rel $v_{1}$. Otherwise, let $\left\{\Gamma_{1}, \ldots, \Gamma_{n}\right\}$ be the collection of innermost subgraphs of $\Gamma$. In either case, let $v_{i}$ be the attaching vertex of $\Gamma_{i}$. Thus if $v_{1}$ is fixed by $\operatorname{TSG}_{+}(\Gamma)$ then for every $i, v_{i}=v_{1}$. Observe that, since $v_{1}$ is an innermost type I vertex, $\Gamma$ has at least two innermost subgraphs and at least two innermost subgraphs rel $v_{1}$. Thus in either case, $n>1$. Furthermore, by the uniqueness of the collection $\left\{\Gamma_{1}, \ldots, \Gamma_{n}\right\}$, each element of $\mathrm{TSG}_{+}(\Gamma)$ takes each $\Gamma_{i}$ to some $\Gamma_{j}$. Furthermore, since $\Gamma_{1}, \ldots, \Gamma_{n}$ are pairwise almost disjoint and $\Gamma_{n}$ is a type I subgraph of $\operatorname{cl}\left(\Gamma-\left(\Gamma_{1} \cup \cdots \cup \Gamma_{n-1}\right)\right)$, we can apply Lemma 1 where $r=n-1$ and $\alpha=\Gamma_{n}$ to obtain a collection of pairwise almost disjoint type I balls, $\left\{B_{1}, \ldots, B_{n}\right\}$ for $\Gamma$, associated with $\left\{\Gamma_{1}, \ldots, \Gamma_{n}\right\}$.

Let $G$ denote the group of all orientation preserving diffeomorphisms of $\left(S^{3}, \Gamma\right)$. Then $G$ induces $\operatorname{TSG}_{+}(\Gamma)$ and for every $a \in \mathrm{TSG}_{+}(\Gamma)$ there is a $g_{a} \in G$ which induces $a$. Let $m$ denote a number larger than the number of vertices in $\Gamma$. We will use the following observation in several places in the proof.

Observation. Suppose that $g:\left(S^{3}, \Gamma_{i}\right) \rightarrow\left(S^{3}, \Gamma_{j}\right)$ is a diffeomorphism such that $g\left(v_{i}\right)=v_{j}$. Let $D_{i}$ and $D_{j}$ be balls containing $\Gamma_{i}$ and $\Gamma_{j}$ respectively such that 
$\partial D_{i} \cap \Gamma_{i}=\left\{v_{i}\right\}$ and $\partial D_{j} \cap \Gamma_{j}=\left\{v_{j}\right\}$. Now $g\left(D_{i}\right)$ and $D_{j}$ are both balls containing $\Gamma_{j}$. Thus the complements of $g\left(D_{i}\right)$ and $D_{j}$ are isotopic by an isotopy of $S^{3}$ fixing $\Gamma_{j}$. Hence $g$ is isotopic to a diffeomorphism $g^{\prime}:\left(S^{3}, \Gamma_{i}\right) \rightarrow\left(S^{3}, \Gamma_{j}\right)$ such that $g^{\prime}\left(D_{i}\right)=D_{j}$ and $g^{\prime}\left|\Gamma_{i}=g\right| \Gamma_{i}$.

Step 1. We prove the proposition in the case where $v_{1}$ is fixed by $\mathrm{TSG}_{+}(\Gamma)$ and some $\Gamma_{i}$ is not setwise invariant under $\mathrm{TSG}_{+}(\Gamma)$.

In this case, $\left\{\Gamma_{1}, \ldots, \Gamma_{n}\right\}$ is the family of innermost subgraphs rel $v_{1}$. Without loss of generality, $\left\{\Gamma_{1}, \ldots, \Gamma_{r}\right\}$ is the orbit of $\Gamma_{1}$, and $r>1$. We define a homomorphism $\Phi: \mathrm{TSG}_{+}(\Gamma) \rightarrow S_{r}$ by letting $\Phi(a)$ be the permutation that $a$ induces on the set $\left\{\Gamma_{1}, \ldots, \Gamma_{r}\right\}$.

To see that $\Phi$ is onto, let $(i j)$ be a non-trivial transposition in $S_{r}$. Since $\Gamma_{i}$ and $\Gamma_{j}$ are both in the orbit of $\Gamma_{1}$, there is some $a \in \mathrm{TSG}_{+}(\Gamma)$ such that $a\left(\Gamma_{i}\right)=\Gamma_{j}$. Now $g_{a}$ is a diffeomorphism of $S^{3}$ such that $g_{a}\left(\Gamma_{i}\right)=\Gamma_{j}$, and $B_{i}$ and $B_{j}$ are balls containing $\Gamma_{i}$ and $\Gamma_{j}$ respectively such that $\partial B_{i} \cap \Gamma_{i}=\left\{v_{1}\right\}$ and $\partial B_{j} \cap \Gamma_{j}=\left\{v_{1}\right\}$. By the Observation, there is a diffeomorphism $g_{a}^{\prime}:\left(S^{3}, \Gamma_{i}\right) \rightarrow\left(S^{3}, \Gamma_{j}\right)$ such that $g_{a}^{\prime}\left(B_{i}\right)=B_{j}$. We will use $g_{a}^{\prime}$ to define a diffeomorphism $g:\left(S^{3}, \Gamma\right) \rightarrow\left(S^{3}, \Gamma\right)$ as follows. Since $B_{i}$ and $B_{j}$ are almost disjoint and $g_{a}^{\prime}$ fixes $v_{1}$, we let $g\left|B_{i}=g_{a}^{\prime}\right| B_{i}$ and $g\left|B_{j}=\left(g_{a}^{\prime}\right)^{-1}\right| B_{j}$. Let $B$ denote a ball containing $B_{i} \cup B_{j}$ such that $\partial B \cap\left(B_{i} \cup B_{j}\right)=$ $\left\{v_{1}\right\}$ and $B \cap \Gamma=\Gamma_{i} \cup \Gamma_{j}$. We can extend $g$ to a diffeomorphism of $B-\left(B_{i} \cup B_{j}\right)$ such that $g \mid \partial B$ is the identity, and then extend $g$ to $S^{3}-B$ by the identity. Now $g$ is an orientation preserving diffeomorphism of $\left(S^{3}, \Gamma\right)$. Let $a^{\prime}$ denote the automorphism induced on $\Gamma$ by $g$. Then $a^{\prime}$ interchanges $\Gamma_{i}$ and $\Gamma_{j}$ and $a^{\prime} \mid \Gamma-\left(\Gamma_{i} \cup \Gamma_{j}\right)$ is the identity. So $\Phi\left(a^{\prime}\right)$ is the transposition $(i j)$. Hence $\Phi$ is onto.

If $\operatorname{ker}(\Phi)$ is trivial, then $\mathrm{TSG}_{+}(\Gamma) \cong S_{r}$ and we are done. So suppose that $N=\operatorname{ker}(\Phi)$ is non-trivial. Let $\Pi$ denote the embedded graph obtained from $\Gamma$ as follows. For each $i \leq r$ such that $\Gamma_{i}$ is not a simple closed curve, we add $i m$ vertices to every edge of $\Gamma_{i}$ containing $v_{1}$. For each $i \leq r$ such that $\Gamma_{i}$ is a simple closed curve we add $i m$ vertices to a single edge of $\Gamma_{i}$. Now each $\Gamma_{i}$ is an innermost subgraph rel $v_{1}$ for $\Pi$, and is the unique innermost subgraph rel $v_{1}$ which contains a chain whose length is between $i m$ and $(i+1) m-1$. Thus every element of $\mathrm{TSG}_{+}(\Pi)$ takes $\Gamma_{i}$ to itself for all $i \leq r$. Now it is not hard to see that $\operatorname{TSG}_{+}(\Pi) \cong \operatorname{ker}(\Phi)$. Finally, let $\Pi^{\prime}$ denote the embedded graph consisting of $r$ edges joined together at a common vertex. Then $\operatorname{TSG}_{+}\left(\Pi^{\prime}\right) \cong S_{r} \cong H / N$. Thus both $N$ and $H / N$ are realizable. So we are done with Step 1.

As a result of Step 1, we assume that one of the following hypotheses holds.

Hypothesis (a). $v_{1}$ is not fixed by $\mathrm{TSG}_{+}(\Gamma)$.

Hypothesis (b). $v_{1}$ is fixed by $\operatorname{TSG}_{+}(\Gamma)$ and every $\Gamma_{i}$ is setwise invariant under $\mathrm{TSG}_{+}(\Gamma)$. 
Step 2. We choose an invariant proper subgraph $\Gamma^{\prime}$ and define a non-trivial homomorphism $\Psi: \mathrm{TSG}_{+}(\Gamma) \rightarrow \mathrm{TSG}_{+}\left(\Gamma^{\prime}\right)$ such that $N=\operatorname{ker}(\Psi)$ is realizable.

First we assume that Hypothesis (a) holds. In this case, $\left\{\Gamma_{1}, \ldots, \Gamma_{n}\right\}$ is the family of innermost subgraphs of $\Gamma$. Without loss of generality, we can assume that the subcollection of innermost subgraphs with attaching vertex $v_{1}$ is $\left\{\Gamma_{1}, \ldots, \Gamma_{t}\right\}$. Let $O$ denote the orbit of $\Gamma_{1} \cup \cdots \cup \Gamma_{t}$ under $\operatorname{TSG}_{+}(\Gamma)$. Without loss of generality there exists an $r \leq n$ such that $O=\Gamma_{1} \cup \cdots \cup \Gamma_{r}$. Now let $\Gamma^{\prime}=\operatorname{cl}(\Gamma-O)$. Then $\Gamma^{\prime}$ is a connected graph. Observe that $\Gamma^{\prime}$ is setwise invariant under $G$, and the homomorphism $\Psi: \mathrm{TSG}_{+}(\Gamma) \rightarrow \mathrm{TSG}_{+}\left(\Gamma^{\prime}\right)$ given by $\Psi(a)=a \mid \Gamma^{\prime}$ for every $a \in \mathrm{TSG}_{+}(\Gamma)$ is not trivial since $v_{1}$ is not fixed by $\mathrm{TSG}_{+}(\Gamma)$.

We create an embedded graph $\Pi$ which realizes $N=\operatorname{ker}(\Psi)$ as follows. Let $\left\{x_{1}, \ldots, x_{s}\right\}$ be distinct vertices representing the orbit of $v_{1}$ under $\operatorname{TSG}_{+}(\Gamma)$. As sets $\left\{x_{1}, \ldots, x_{s}\right\}=\left\{v_{1}, \ldots, v_{r}\right\}$, however we may have $r>s$ if some $v_{i}=v_{j}$ for $i \neq j$. Let $\beta_{1}, \ldots, \beta_{s+1}$ be arcs such that each $\beta_{j}$ contains $j m$ vertices in its interior. We obtain $\Pi$ from $\Gamma_{1} \cup \ldots \cup \Gamma_{r}$ by adding $\beta_{1}, \ldots, \beta_{s+1}$ on the outside of $B_{1}, \ldots, B_{r}$, such that $\beta_{1}$ is attached to $x_{1}$; for each $j$ such that $1<j<s+1, \beta_{j}$ has one endpoint at $x_{j}$ and the other endpoint at $x_{j+1}$; and $\beta_{s+1}$ is attached to $x_{s}$. For each $j, \beta_{j}$ is the only chain in $\Pi$ of length $j m$, and hence each $\beta_{j}$ is invariant under $\mathrm{TSG}_{+}(\Pi)$. Thus for each $j \leq s,\left\{\Gamma_{i} \mid v_{i}=x_{j}\right\}$ is setwise invariant under $\operatorname{TSG}_{+}(\Pi)$. Now it is not hard to check that $\mathrm{TSG}_{+}(\Pi) \cong \operatorname{ker}(\Psi)$.

Now suppose that Hypothesis (b) holds. Then $\left\{\Gamma_{1}, \ldots, \Gamma_{n}\right\}$ is the family of innermost subgraphs of $\Gamma$ rel $v_{1}$. Since $\operatorname{TSG}_{+}(\Gamma)$ is non-trivial, without loss of generality there is some $a \in \mathrm{TSG}_{+}(\Gamma)$ which induces a non-trivial automorphism on $\Gamma_{n}$. In this case we let $\Gamma^{\prime}=\Gamma_{n}$. Now $\Gamma^{\prime}$ is setwise invariant under $G$, and again $\Psi: \mathrm{TSG}_{+}(\Gamma) \rightarrow \mathrm{TSG}_{+}\left(\Gamma^{\prime}\right)$ defined by $\Psi(a)=a \mid \Gamma^{\prime}$ for every $a \in \mathrm{TSG}_{+}(\Gamma)$ is non-trivial. In this case, we let $\Pi=\Gamma_{1} \cup \cdots \cup \Gamma_{n-1}$ together with two arcs added at $v_{1}$, one with $m$ vertices and the other with $2 m$ vertices (this guarantees that $\Pi$ is not a single arc). Then every element of $\mathrm{TSG}_{+}(\Pi)$ fixes $v_{1}$, and we see that $\Pi$ realizes $N=\operatorname{ker}(\Psi)$. So we are done with Step 2 .

Next we will introduce some notation that will be used throughout the rest of the proof. If Hypothesis (a) holds, then $v_{1}$ is not fixed by $\operatorname{TSG}_{+}(\Gamma)$. Hence there is some innermost subgraph rel $v_{1}$ which is not one of the $\Gamma_{i}$. By our choice of $v_{1}$, at most one innermost subgraph rel $v_{1}$ is not one of the $\Gamma_{i}$. Thus $\delta=\operatorname{cl}\left(\Gamma-\left(\Gamma_{1} \cup \cdots \cup \Gamma_{t}\right)\right)$ is an innermost subgraph rel $v_{1}$. Also $\Gamma^{\prime}=\operatorname{cl}\left(\Gamma-\left(\Gamma_{1} \cup \cdots \cup \Gamma_{r}\right)\right) \subset \delta$. If Hypothesis (b) holds, then we let $t=r=n-1$, and again let $\delta=\operatorname{cl}\left(\Gamma-\left(\Gamma_{1} \cup \cdots \cup \Gamma_{t}\right)\right)$. In this case $\delta=\Gamma_{n}=\Gamma^{\prime}$, and hence again $\delta$ is an innermost subgraph rel $v_{1}$. We will use $r$ in the next step and use $t$ and $\delta$ in Step 5.

In general, $\Psi: \mathrm{TSG}_{+}(\Gamma) \rightarrow \mathrm{TSG}_{+}\left(\Gamma^{\prime}\right)$ may not be surjective. In Step 3 we will create a new embedded graph $\Lambda$ by adding vertices or chains of vertices to $\Gamma^{\prime}$, and in Step 4 we will show that $\Lambda$ realizes $H / N$. 
Step 3. We construct an embedded graph $\Lambda$ which contains $\Gamma^{\prime}$ and satisfies the following conditions:

(1) For every $a \in \mathrm{TSG}_{+}(\Gamma)$, there is a $g_{a} \in G$ such that $g_{a}(\Lambda)=\Lambda$.

(2) If $g, h \in G$ induce the same automorphism of $\Gamma$ and leave $\Lambda$ setwise invariant, then $g$ and $h$ induce the same automorphism of $\Lambda$.

(3) If $g$ is a diffeomorphism of $\left(S^{3}, \Lambda\right)$, then $g\left(\left\{v_{1}, \ldots, v_{r}\right\}\right)=\left\{v_{1}, \ldots, v_{r}\right\}$ and $g\left(\Gamma^{\prime}\right)=\Gamma^{\prime}$.

Case 1. $v_{1}$ has valence one in $\Gamma^{\prime}$.

Then $\Gamma^{\prime}$ contains a chain $P$ with one endpoint at $v_{1}$. Suppose that $\Gamma^{\prime}=P$, then $\mathrm{TSG}_{+}\left(\Gamma^{\prime}\right) \cong \mathbb{Z}_{2}$. Since there is some $a \in \mathrm{TSG}_{+}(\Gamma)$ which induces a non-trivial automorphism on $\Gamma^{\prime}, \operatorname{im}(\Psi)=\mathrm{TSG}_{+}\left(\Gamma^{\prime}\right)$. Thus $H / N$ is realizable. If $N$ is trivial, then $\mathrm{TSG}_{+}(\Gamma) \cong \mathbb{Z}_{2}$, contrary to our initial assumption. If $N$ is non-trivial we would be done with the proposition. Thus we assume that $\Gamma^{\prime} \neq P$.

Let $\varepsilon$ be the edge in $P$ containing $v_{1}$. We create $\Lambda$ from $\Gamma^{\prime}$ by adding $m$ vertices to each edge in the orbit of $\varepsilon$ under $\mathrm{TSG}_{+}(\Gamma)$. It is easy to see that Conditions (1) and (2) are satisfied for $\Lambda$. We abuse notation and let $P$ denote both the chain in $\Gamma^{\prime}$ containing $v_{1}$ and the chain in $\Lambda$ containing $v_{1}$. Since $P$ and its orbit under $\operatorname{TSG}_{+}(\Gamma)$ are the only chains of length at least $m$ in $\Lambda$, the orbit of $P$ under $\operatorname{TSG}_{+}(\Lambda)$ is the same as the orbit of $P$ under $\mathrm{TSG}_{+}(\Gamma)$. Since $\Gamma^{\prime} \neq P$, one endpoint of $P$ has valence at least three in $\Lambda$. Hence no element of $\mathrm{TSG}_{+}(\Lambda)$ interchanges the endpoints of $P$ or any chain in its orbit. Thus for any diffeomorphism $g:\left(S^{3}, \Lambda\right) \rightarrow\left(S^{3}, \Lambda\right)$, we must have $g\left(\left\{v_{1}, \ldots, v_{r}\right\}\right)=\left\{v_{1}, \ldots, v_{r}\right\}$, and $g\left(\Gamma^{\prime}\right)=\Gamma^{\prime}$. Thus Condition (3) is satisfied.

Case 2. The valence of $v_{1}$ is at least two in $\Gamma^{\prime}$.

Consider vertex neighborhoods $N\left(v_{1}\right), \ldots, N\left(v_{r}\right)$. For each $i \leqslant r$ and each edge $\varepsilon_{j}$ in $\Gamma^{\prime}$ which contains $v_{i}$, let $f_{j}=N\left(v_{i}\right) \cap \varepsilon_{j}$ and let $w_{j}$ be the point where $f_{j}$ meets $\partial N\left(v_{i}\right)$. If Hypothesis (a) is satisfied, choose a collection of pairwise almost disjoint regular neighborhoods $N\left(f_{j}\right)$ in $N\left(v_{i}\right)-\left(B_{1} \cup \cdots \cup B_{r}\right)$. If Hypothesis (b) is satisfied, choose a collection of pairwise almost disjoint regular neighborhoods $N\left(f_{j}\right)$ in $N\left(v_{i}\right) \cap B_{n}$. In either case, for each $j$, we let $d_{j} \subset N\left(f_{j}\right)$ be an arc with endpoints $v_{i}$ and $w_{j}$ such that $d_{j} \cup f_{j}$ bounds a disk $E_{j}$ in $N\left(f_{j}\right)$. We create $\Lambda$ from $\Gamma^{\prime}$ by adding each $d_{j}$ together with the vertex $w_{j}$ and $m$ additional vertices on $d_{j}$.

As an arc, $d_{j}$ is isotopic to $f_{j}$ fixing $\Gamma$. Hence for every $a \in \mathrm{TSG}_{+}(\Gamma)$, we can choose $g_{a} \in G$ such that $g_{a}(\Lambda)=\Lambda$. Hence Condition (1) is satisfied. Furthermore, by our construction of $\Lambda$, Condition (2) must also be satisfied. Observe that since the valence of $v_{1}$ is at least two in $\Gamma^{\prime}, \Lambda$ has at least two $d_{j}$ 's containing the vertex $v_{1}$. Thus for each $i \leq r, v_{i}$ has valence at least four in $\Lambda$, while each $w_{i}$ has valence three. Therefore, $v_{1}, \ldots, v_{r}$ are the only vertices of valence more than three in $\Lambda$ which are endpoints of chains of length $m$. It follows that for any diffeomorphism 
$g:\left(S^{3}, \Lambda\right) \rightarrow\left(S^{3}, \Lambda\right)$, we must have $g\left(\left\{v_{1}, \ldots, v_{r}\right\}\right)=\left\{v_{1}, \ldots, v_{r}\right\}$ and $g\left(\Gamma^{\prime}\right)=$ $\Gamma^{\prime}$. Hence Condition (3) is satisfied. Thus we are done with Step 3.

Step 4. We prove that $H / N$ is realized by $\Lambda$.

By Conditions (1) and (2), we can define a homomorphism $\Phi: \mathrm{TSG}_{+}(\Gamma) \rightarrow$ $\mathrm{TSG}_{+}(\Lambda)$ by letting $\Phi(a)$ denote the automorphism that $g_{a}$ induces on $\Lambda$. Furthermore, it is easy to check that $\operatorname{ker}(\Phi)=\operatorname{ker}(\Psi)=N$.

To show that $\Phi$ is onto, we let $a \in \mathrm{TSG}_{+}(\Lambda)$. By definition there is an orientation preserving diffeomorphism $g:\left(S^{3}, \Lambda\right) \rightarrow\left(S^{3}, \Lambda\right)$ inducing $a$ on $\Lambda$. We will construct an orientation preserving diffeomorphism $h:\left(S^{3}, \Gamma\right) \rightarrow\left(S^{3}, \Gamma\right)$ that leaves $\Lambda$ invariant and induces $a$ on $\Lambda$ as follows.

Case 1. $\Gamma$ satisfies Hypothesis (a).

Since $\left\{v_{1}, \ldots, v_{r}\right\}$ is the orbit of $v_{1}$ under $G$ and by Condition $(3) g\left(\left\{v_{1}, \ldots, v_{r}\right\}\right)=$ $\left\{v_{1}, \ldots, v_{r}\right\}$, for every $i \leq r$, there is a $g_{i} \in G$ such that $g_{i}\left(v_{i}\right)=g\left(v_{i}\right)$. Now for each $i \leq r$, we can choose $j(i) \leq r$ such that $g_{i}\left(\Gamma_{i}\right)=\Gamma_{j(i)}$, and hence $g_{i}\left(v_{i}\right)=v_{j(i)}$. It follows from the Observation that for each $i \leq r$, there is an orientation preserving diffeomorphism $g_{i}^{\prime}:\left(B_{i}, \Gamma_{i}\right) \rightarrow\left(B_{j(i)}, \Gamma_{j(i)}\right)$. Since $B_{1}, \ldots, B_{r}$ are pairwise almost disjoint, for each $i \leq r$, we can define $h \mid B_{i}=g_{i}^{\prime}$. Then $h\left(v_{i}\right)=g_{i}^{\prime}\left(v_{i}\right)=v_{j(i)}=g\left(v_{i}\right)$.

Next we define a collection $\left\{D_{1}, \ldots, D_{r}\right\}$ of pairwise almost disjoint innermost balls for $\Gamma$ with associated subgraphs $\left\{\Gamma_{1}, \ldots, \Gamma_{r}\right\}$ such that each $B_{i}-\left\{v_{i}\right\} \subset \operatorname{int}\left(D_{i}\right)$ and $D_{i} \cap \Lambda=B_{i} \cap \Lambda=\left\{v_{i}\right\}$. Since each $g\left(v_{i}\right)=v_{j(i)}$, each $g\left(D_{i}\right)$ is isotopic to $D_{j(i)}$ by an isotopy of $S^{3}$ fixing $\Lambda$. As in the Observation, it follows that $g$ is isotopic to an orientation preserving diffeomorphism $g^{\prime}:\left(S^{3}, \Lambda\right) \rightarrow\left(S^{3}, \Lambda\right)$ such that $g^{\prime}|\Lambda=g| \Lambda$ and for each $i \leq r, g^{\prime}\left(D_{i}\right)=D_{j(i)}$. Hence $g^{\prime}\left(\left\{v_{1}, \ldots, v_{r}\right\}\right)=\left\{v_{1}, \ldots, v_{r}\right\}$ and $g^{\prime}\left(\Gamma^{\prime}\right)=\Gamma^{\prime}$. Thus we define $h\left|\operatorname{cl}\left(S^{3}-\left(D_{1} \cup \cdots \cup D_{r}\right)\right)=g^{\prime}\right| \operatorname{cl}\left(S^{3}-\left(D_{1} \cup \cdots \cup D_{r}\right)\right)$.

Finally, for every $i \leq r$, since $h\left(\partial D_{i}\right)=\partial D_{j(i)}$ and $h\left(\partial B_{i}\right)=\partial B_{j(i)}$, and $h \mid \partial D_{i}$ and $h \mid \partial B_{i}$ are both orientation preserving diffeomorphisms taking $v_{i}$ to $v_{j(i)}$, we can extend $h$ to a diffeomorphism from $D_{i}-B_{i}$ to $D_{j(i)}-B_{j(i)}$. Now $h$ is a diffeomorphism of $\left(S^{3}, \Lambda\right)$ which induces $a$ on $\Lambda$ and $h(\Gamma)=\Gamma$. Let $b$ be the automorphism which $h$ induces on $\Gamma$. Then $\Phi(b)=a$, and hence $\Phi$ is onto.

Case 2. $\Gamma$ satisfies Hypothesis (b).

In this case, $\Gamma^{\prime}=\Gamma_{n}$, we have $\Lambda \subset B_{n}$ and $\partial B_{n} \cap \Lambda=\left\{v_{1}\right\}$. Now by the Observation, since $g(\Lambda)=\Lambda$ and $g\left(v_{1}\right)=v_{1}, g$ is isotopic to an orientation preserving diffeomorphism $g^{\prime}:\left(S^{3}, \Lambda\right) \rightarrow\left(S^{3}, \Lambda\right)$ such that $g^{\prime}\left(B_{n}\right)=B_{n}$ and $g^{\prime}|\Lambda=g| \Lambda$. Define $h\left|B_{n}=g^{\prime}\right| B_{n}$. Let $D_{n}$ be an innermost ball for $\Gamma_{n}$ such that $B_{n}-\left\{v_{n}\right\} \subset \operatorname{int}\left(D_{n}\right)$. Since $h \mid \partial B_{n}$ is orientation preserving we can extend $h$ to a diffeomorphism of $D_{n}-B_{n}$ such that $h \mid \partial D_{n}$ is the identity, and then define $h$ to be the identity on $S^{3}-D_{n}$. Now $h$ is a diffeomorphism of $\left(S^{3}, \Lambda\right)$ which induces $a$ on $\Lambda$, and $h(\Gamma)=\Gamma$. So as above, 
let $b$ be the automorphism which $h$ induces on $\Gamma$, then $\Phi(b)=a$. Thus again $\Phi$ is onto.

Therefore in either case, $H / N \cong \mathrm{TSG}_{+}(\Lambda)$. Thus we are done with Step 4 .

In Step 2 we saw that $N=\operatorname{ker}(\Psi)$ is realizable. If $N$ is non-trivial then we are done. Otherwise, $H$ is realized by $\Lambda$. Hence we will be done after we prove the following.

Step 5. We show that $\Lambda$ has fewer type I vertices than $\Gamma$.

First suppose that $\Lambda$ was created in Case 1 of Step 3. Then $\Lambda$ is homeomorphic as a topological space to $\Gamma^{\prime}$. Recall that a type I vertex must have valence at least three. So, $\Gamma^{\prime}$ and $\Lambda$ have the same set of type I vertices, and by Lemma 1 , every type I vertex of $\Gamma^{\prime}$ is a type I vertex of $\Gamma$. On the other hand, $v_{1}$ is a type I vertex of $\Gamma$. However, since the valence of $v_{1}$ is one in $\Lambda, v_{1}$ is not a type I vertex of $\Lambda$. Therefore, $\Lambda$ has fewer type I vertices than $\Gamma$.

Thus we can assume that $\Lambda$ was created in Case 2 of Step 3. Let $x$ be a type I vertex of $\Lambda$. We will show that $x$ is a type I vertex of $\Gamma$ which is not one of $v_{1}, \ldots, v_{r}$. Let $\alpha$ be a type I subgraph of $\Lambda$ with attaching vertex $x$, and let $\alpha^{\prime}=\operatorname{cl}(\Lambda-\alpha)$. For each $j$, the simple closed curve $f_{j} \cup d_{j}$ is contained entirely in either $\alpha$ or $\alpha^{\prime}$. Hence $\alpha$ and $\alpha^{\prime}$ each contain at least one edge of $\Gamma^{\prime}$. Let $\Theta=\Lambda \cup\left(\Gamma_{1} \cup \cdots \cup \Gamma_{r}\right)$. Then $\Gamma_{1}, \ldots, \Gamma_{r}$ are type I subgraphs of $\Theta$. Thus by Lemma $1, \Theta$ has a type I ball $E$ with attaching vertex $x$ such that $E \cap \Lambda=\alpha$ and $E$ is almost disjoint from each $\Gamma_{i}$ such that $v_{i} \in \operatorname{cl}\left(\Gamma^{\prime}-\alpha\right)$. Then by definition, $E$ is a type I ball for $\Lambda$ associated with $\alpha$. Let $\Sigma=\partial E$.

Now suppose that $x$ is a vertex of $\Gamma^{\prime}$. Then either the valence of $x$ is at least three in $\Gamma^{\prime}$ or $x=v_{i}$ for some $i$. In either case, the valence of $x$ is at least three in $\Gamma$. Also $\Sigma \cap \Gamma \subset \Sigma \cap \Theta=\{x\}$, and each component of $\operatorname{cl}\left(S^{3}-\Sigma\right)$ contains at least one edge of $\Gamma$. Hence $\Sigma$ is a type I sphere for $\Gamma$ and $E \cap \Gamma$ is a type I subgraph of $\Gamma$ with attaching vertex $x$.

Recall from the end of Step 2 that $\Gamma_{1}, \ldots, \Gamma_{t}$ all have attaching vertex $v_{1}$, and $\delta=\operatorname{cl}\left(\Gamma-\left(\Gamma_{1} \cup \cdots \cup \Gamma_{t}\right)\right)$ is an innermost subgraph rel $v_{1}$ containing $\Gamma^{\prime}$. Suppose for the sake of contradiction that $x=v_{1}$. For all $i \leq t, v_{i}=x \in \operatorname{cl}\left(\Gamma^{\prime}-\alpha\right)$, hence $E$ is almost disjoint from $\Gamma_{1} \cup \cdots \cup \Gamma_{t}$. Also since $E \cap \Gamma$ is a type I subgraph of $\Gamma$ with attaching vertex $v_{1}, \Gamma$ has an innermost subgraph rel $v_{1}$ which is contained in $E \cap \Gamma$. Let $\beta$ be this subgraph. Then $\beta \subset E \cap \Gamma \subset \delta$. However, $\beta \subset \delta$ implies that $\beta=\delta$, since $\beta$ and $\delta$ are both innermost subgraphs rel $v_{1}$. Thus $\Gamma^{\prime} \subset \delta=E \cap \Gamma$. But $\alpha^{\prime} \subset$ $\operatorname{cl}\left(S^{3}-E\right)$ contains at least one edge of $\Gamma^{\prime}$. This contradiction implies that $x \neq v_{1}$. Furthermore, for each $i \leqslant r$, there is a diffeomorphism $g:\left(S^{3}, \Lambda\right) \rightarrow\left(S^{3}, \Lambda\right)$ such that $g\left(v_{1}\right)=v_{i}$. Thus, for all $i \leqslant r, x \neq v_{i}$. Hence we have shown that if $x$ is a vertex of $\Gamma^{\prime}$, then $x$ is a type I vertex of $\Gamma$ which is not one of $v_{1}, \ldots, v_{r}$.

Finally, for the sake of contradiction, suppose that $x$ is not a vertex of $\Gamma^{\prime}$. Since the valence of $x$ must be at least three in $\Lambda, x=w_{j}$ for some $j$. Without loss of 
generality assume that $f_{j} \cup d_{j} \subset \alpha^{\prime}$ and hence $\varepsilon_{j}-f_{j} \subset \alpha$. For some $i \leqslant r, v_{i}$ is an endpoint of $d_{j}$. We will show that our assumption that $w_{j}$ is a type I vertex of $\Lambda$ implies that $v_{i}$ is also a type I vertex of $\Lambda$. Now $v_{i}$ has valence at least four in $\Lambda$ and all of the edges of $\Lambda$ containing $v_{i}$ are in $\alpha^{\prime}$. By our construction of $\Lambda, f_{j} \cup d_{j}$ bounds a disk $E_{j}$ such that $\operatorname{int}\left(E_{j}\right)$ is disjoint from $\Lambda$. Thus by taking the union of $E$ and a ball containing $E_{j}$ we obtain a type I ball $B$ for $\Lambda$ with attaching vertex $v_{i}$ such that $B \cap \Lambda=\alpha \cup f_{j} \cup d_{j}$. Thus $v_{i}$ is a type I vertex of $\Lambda$. But we saw above that this is impossible. Hence $x \neq w_{j}$ for any $j$.

Thus we have shown that every type I vertex of $\Lambda$ is a type I vertex of $\Gamma$ which is not one of the vertices $v_{1}, \ldots, v_{r}$. Hence the set of type I vertices of $\Lambda$ is a proper subset of the type I vertices of $\Gamma$. This completes the proof of Proposition 2.

\section{Embedding graphs in $S^{3}$}

In this section we prove the converse of Theorem 2. In particular, in Theorem 3 we will show that for every finite subgroup $G$ of $\operatorname{Diff}_{+}\left(S^{3}\right)$, there is an embedded complete bipartite graph $\Gamma$ with $\mathrm{TSG}_{+}(\Gamma) \cong G$. A complete bipartite graph $K_{n, n}$ is the graph consisting of two sets of $n$ vertices $V$ and $W$ together with edges joining every vertex in $V$ to every vertex in $W$.

Our strategy to construct $\Gamma$ will be as follows. Let $n$ denote the order of $G$. Then the sets of vertices $V$ and $W$ will be embedded as the orbits under $G$ of distinct points in $S^{3}$. We will embed the edges of $K_{n, n}$ by lifting paths from the orbit space $S^{3} / G$. Finally, by tying distinct knots in edges from different orbits we will ensure that $\mathrm{TSG}_{+}(\Gamma)$ is not larger than $G$.

We will use the following terminology. An edge $e$ of a graph $\gamma$ is said to be invertible if there exists some $a \in \operatorname{Aut}(\gamma)$ that interchanges the vertices of $e$. In this case we say that $a$ inverts $e$. Analogously if $\varepsilon$ is an edge in an embedded graph $\Gamma$, and there is some diffeomorphism $g$ of $\left(S^{3}, \Gamma\right)$ such that $g$ interchanges the vertices of $\varepsilon$, then we say $\varepsilon$ is invertible and $g$ inverts $\varepsilon$.

Graph Embedding Lemma. Let $\gamma$ be a graph. Let $H$ be a subgroup of Aut $(\gamma)$ that is isomorphic to a finite subgroup $G$ of $\operatorname{Diff}_{+}\left(S^{3}\right)$. Suppose that no non-trivial element of $H$ fixes any vertex or inverts any edge of $\gamma$. Then there is an embedded graph $\Gamma$ with underlying abstract graph $\gamma$ such that $G$ induces $H$ on $\Gamma$.

Proof. Smith $[\mathrm{Sm}]$ has shown that the fixed point set of every finite order orientation preserving homeomorphism of $S^{3}$ is either the empty set or a simple closed curve. Let $Y$ denote the union of the fixed point sets of all of the non-trivial elements of $G$. Then $Y$ is the union of finitely many simple closed curves whose pairwise intersection consists of finitely many points. So $M=S^{3}-Y$ is path connected and setwise invariant under $G$. 
Let $\Psi: H \rightarrow G$ be an isomorphism and for each $a \in H$, define $g_{a}=\Psi(a)$. Let $\left\{w_{1}, \ldots, w_{q}\right\}$ be a set consisting of one representative from each vertex orbit of $\gamma$ under $H$. Let $v_{1}, \ldots, v_{q}$ be distinct points in $M$ which have disjoint orbits under $G$. For each $i \leqslant q$, we embed the vertex $w_{i}$ as the point $v_{i}$.

We embed an arbitrary vertex $w$ of $\gamma$ as follows. Since no vertex of $\gamma$ is fixed by any non-trivial element of $H$, there is a unique $a \in H$ and $i \leqslant q$ such that $w=a\left(w_{i}\right)$. Thus every $w$ determines a unique point $g_{a}\left(v_{i}\right)$ in $M$. So we embed $w$ as $g_{a}\left(v_{i}\right)$. Let $V$ denote the set of thus embedded vertices; then $G$ leaves $V$ setwise invariant. Since $v_{1}, \ldots, v_{q}$ are in $M$ and have disjoint orbits under $G$, all of the vertices of $\gamma$ are embedded as distinct points in $M$. The map sending each $a \in H$ to $g_{a} \mid V$ is an isomorphism, since $\Psi$ is an isomorphism and $V$ is disjoint from $Y$. Thus $G$ induces $H$ on the set $V$.

Let $\left\{e_{1}, \ldots, e_{n}\right\}$ be a set consisting of one representative from each edge orbit of $\gamma$ under $H$. For each $i$, let $x_{i}$ and $y_{i}$ be the embedded vertices of $e_{i}$. Since $M$ is path connected, for each $i$ there is a path $\alpha_{i}$ in $M$ from $x_{i}$ to $y_{i}$.

Let $\pi: M \rightarrow M / G$ denote the quotient map. Then $\pi$ is a covering map, and the quotient space $Q=M / G$ is a 3-manifold. For each $i$, let $\alpha_{i}^{\prime}=\pi \circ \alpha_{i}$. Then $\alpha_{i}^{\prime}$ is a path or loop from $\pi\left(x_{i}\right)$ to $\pi\left(y_{i}\right)$. Using a general position argument in $Q$, we can homotop each $\alpha_{i}^{\prime}$, fixing its endpoints, to a simple path or loop $\rho_{i}^{\prime}$ such that $\operatorname{int}\left(\rho_{1}^{\prime}(I)\right), \ldots, \operatorname{int}\left(\rho_{n}^{\prime}(I)\right)$, and $\pi(V)$ are pairwise disjoint. For each $i$, let $\rho_{i}$ be the lift of $\rho_{i}^{\prime}$ beginning at $x_{i}$. Since $\rho_{i}^{\prime}=\pi \circ \rho_{i}$ is one-to-one except possibly on the set $\{0,1\}$, we know that $\rho_{i}$ must also be one-to-one except possibly on the set $\{0,1\}$. Since $\rho_{i}^{\prime}$ is homotopic to $\alpha_{i}^{\prime}$ fixing its endpoints, $\rho_{i}$ is also homotopic to $\alpha_{i}$ fixing its endpoints. Thus $\rho_{i}$ is a simple path in $M$ from $x_{i}$ to $y_{i}$.

We embed each $e_{i}$ as $\rho_{i}(I)$. We embed an arbitrary edge $e$ as follows. Observe that since no non-trivial element of $H$ fixes any vertex or inverts any edge, no edge is setwise invariant under any non-trivial element of $H$. Thus there are unique $a \in H$ and $i \leq n$ such that $e=a\left(e_{i}\right)$. Hence $e$ determines a unique $\operatorname{arc} g_{a}\left(\rho_{i}(I)\right)$ in $M$ from $g_{a}\left(x_{i}\right)$ to $g_{a}\left(y_{i}\right)$. So we embed $e$ as $\varepsilon=g_{a}\left(\rho_{i}(I)\right)$. Let $E$ denote the set of thus embedded edges. Then $G$ leaves $E$ setwise invariant.

Let $\Gamma$ consist of the vertices $V$ together with the embedded edges $E$. Then $\Gamma$ is setwise invariant under $G$. We see that $\Gamma$ is an embedded graph as follows. First, since each $\operatorname{int}\left(\rho_{i}^{\prime}(I)\right)$ is disjoint from $\pi(V)$, each embedded edge must be disjoint from $V$. Similarly, since for $i \neq j, \rho_{i}^{\prime}(I)$ and $\rho_{j}^{\prime}(I)$ have disjoint interiors, for every $g, h \in G, g\left(\rho_{i}(I)\right)$ and $h\left(\rho_{j}(I)\right)$ have disjoint interiors. Finally, since $\rho_{i}^{\prime}$ is a simple path or loop, if $g \neq h$, then $g\left(\rho_{i}(I)\right)$ and $h\left(\rho_{i}(I)\right)$ have disjoint interiors.

Hence $\Gamma$ is an embedded graph with underlying abstract graph $\gamma$ such that $G$ induces $H$ on $\Gamma$.

In the proof of Proposition 4, we would like to be able to add a knot $\kappa$ to a particular edge $\varepsilon$ of $\Gamma$ so that no element of $\mathrm{TSG}_{+}(\Gamma)$ can take $\varepsilon$ to an edge which does not 
also contain $\kappa$. Furthermore, we would like to be able to add a non-invertible knot to an edge $\varepsilon$ so that no element of $\mathrm{TSG}_{+}(\Gamma)$ can invert $\varepsilon$.

We will use the concept of local knotting. Let $\Gamma$ be an embedded graph, let $\kappa$ be a knot type, and let $\varepsilon$ be some edge which is contained in a simple closed curve in $\Gamma$. We say that $\varepsilon$ contains the local knot $\kappa$ with ball $B$, if $B$ is a ball and $B \cap \Gamma$ is an $\operatorname{arc} \alpha$ in int $(\varepsilon)$ such that $\alpha$ is properly embedded in $B$ and the union of $\alpha$ and an arc in $\partial B$ has knot type $\kappa$. When we say an embedded graph $\Gamma^{\prime}$ is obtained from $\Gamma$ by adding $\kappa$ to $\varepsilon$ with ball $B$ we will mean that $\Gamma^{\prime}$ is obtained from $\Gamma$ by replacing an $\operatorname{arc} \alpha$ in int $(\varepsilon)$ with an $\operatorname{arc} \alpha^{\prime}$ in a regular neighborhood $B$ of $\alpha$ which is disjoint from $\Gamma-\alpha$ such that $\alpha^{\prime}$ is properly embedded in $B$, and the union of $\alpha^{\prime}$ and an arc in $\partial B$ has knot type $\kappa$.

Suppose we add a local prime knot $\kappa$ to an edge $\varepsilon$ of $\Gamma$ and call the new embedded edge $\varepsilon^{\prime}$. We see as follows that if $\kappa^{\prime} \neq \kappa$ is a prime knot that was not a local knot of $\varepsilon$, then $\kappa^{\prime}$ is not a local knot of $\varepsilon^{\prime}$. Suppose for the sake of contradiction that $\varepsilon^{\prime}$ contains $\kappa^{\prime}$ with ball $B^{\prime}$. Since the balls $B$ and $B^{\prime}$ for $\kappa$ and $\kappa^{\prime}$ are disjoint from $\Gamma-\varepsilon$, and $\varepsilon$ is contained in a simple closed curve $C$ in $\Gamma$, it is enough to prove the assertion in the case when $\Gamma=C$. But in this case the assertion follows immediately from the prime decomposition theorem for knots.

Orienting an edge from one endpoint to the other naturally induces an orientation on any local knot contained in the edge. If $\kappa$ is a non-invertible prime knot not contained in the oriented edge $\varepsilon$, then, by an argument similar to the above paragraph, adding $\kappa$ to $\varepsilon$ to get $\varepsilon^{\prime}$ does not result in the oriented edge $\varepsilon^{\prime}$ containing the reverse of $\kappa$. In particular, this means that if $\Gamma^{\prime}$ is the graph obtained from $\Gamma$ by adding $\kappa$ to $\varepsilon$, then there is no $a \in \mathrm{TSG}_{+}\left(\Gamma^{\prime}\right)$ which inverts $\varepsilon^{\prime}$.

We want to be able to add a new local knot to one edge of an embedded graph without causing other edges to contain that local knot. Let $\varepsilon_{1}$ and $\varepsilon_{2}$ be edges of $\Gamma$. A bridging sphere between $\varepsilon_{1}$ and $\varepsilon_{2}$ is a sphere $S$ which meets $\Gamma$ transversely in $\left\{x_{1}, x_{2}\right\}$, where each $x_{i}$ is a point in the interior of $\varepsilon_{i}$.

Local Knotting Lemma. Let $\Gamma$ be an embedded graph with distinct edges $\varepsilon_{1}$ and $\varepsilon_{2}$, each contained in a simple closed curve in $\Gamma$, such that there is no bridging sphere between $\varepsilon_{1}$ and $\varepsilon_{2}$. Let $\kappa_{1}$ and $\kappa_{2}$ be (not necessarily distinct) knot types such that $\varepsilon_{2}$ does not contain the local knot $\kappa_{2}$. Then adding $\kappa_{1}$ to $\varepsilon_{1}$ does not make $\varepsilon_{2}$ contain the local knot $\kappa_{2}$.

Proof. Let $\Gamma^{\prime}$ be the graph obtained from $\Gamma$ by adding the local knot $\kappa_{1}$ to $\varepsilon_{1}$ with ball $B_{1}$, and let $\varepsilon_{1}^{\prime}$ denote the edge in $\Gamma^{\prime}$ obtained from $\varepsilon_{1}$ in this way.

Suppose that in $\Gamma^{\prime}, \varepsilon_{2}$ contains the local knot $\kappa_{2}$ with ball $B_{2}$. By general position, we can assume that $\partial B_{1}$ and $\partial B_{2}$ intersect in a collection of pairwise disjoint circles. Suppose there is a circle of intersection that bounds a disk $F$ on $\partial B_{1}$ such that $F$ is disjoint from $\Gamma^{\prime}$. Choose $C$ to be an innermost circle of intersection of $\partial B_{2}$ with 
$F$, and let $D_{1}$ be the disk in $F$ which is bounded by $C$. Suppose, for the sake of contradiction, that some component $D_{2}$ of $\partial B_{2}-C$ contains precisely one point of $\varepsilon_{2}$. By the hypotheses of the lemma, $\varepsilon_{2}$ is contained in a simple closed curve in $\Gamma^{\prime}$. Now the sphere $D_{1} \cup D_{2}$ meets this simple closed curve transversely in a single point, which is impossible. Thus $C$ bounds a disk $D_{2}$ on $\partial B_{2}$ which is disjoint from $\Gamma^{\prime}$. Then the sphere $D_{1} \cup D_{2}$ is disjoint from $\Gamma^{\prime}$, so it bounds a ball $X$ which is also disjoint from $\Gamma^{\prime}$. Therefore, while fixing $\Gamma^{\prime}$, we can isotop $D_{2}$ through $X$ to a disk just past $D_{1}$, and thus eliminate the circle of intersection $C$. By repeating this process as necessary, we can isotop $B_{2}$, fixing $\Gamma^{\prime}$, to a new ball $B_{2}^{\prime}$ such that no circle of $\partial B_{1} \cap \partial B_{2}^{\prime}$ bounds a disk on $\partial B_{1}$ disjoint from $\Gamma^{\prime}$. Since $B_{2}^{\prime}$ is isotopic to $B_{2}$ fixing $\Gamma^{\prime}, B_{2}^{\prime}$ is also a ball for the local knot $\kappa_{2}$ in $\varepsilon_{2}$.

Suppose, for the sake of contradiction, that $\partial B_{1} \cap \partial B_{2}^{\prime}$ is empty. Then $B_{1}$ and $B_{2}^{\prime}$ are disjoint. Hence we can replace $\varepsilon_{1}^{\prime} \cap B_{1}$ by the unknotted arc $\varepsilon_{1} \cap B_{1}$ without introducing any intersections between $\varepsilon_{1}$ and $B_{2}^{\prime}$. Thus, in $\Gamma, \varepsilon_{2}$ contains the local knot $\kappa_{2}$ with ball $B_{2}^{\prime}$, which is contrary to the hypothesis of our lemma. Therefore $\partial B_{1} \cap \partial B_{2}^{\prime}$ must contain one or more circles of intersection, none of which bounds a disk on $\partial B_{1}$ which is disjoint from $\Gamma^{\prime}$. Let $C$ be a circle of intersection of $\partial B_{1} \cap \partial B_{2}^{\prime}$ that bounds an innermost disk $D_{1}$ on $\partial B_{1}$. Let $D_{2}$ be a disk bounded by $C$ on $\partial B_{2}^{\prime}$. Now $\varepsilon_{1}^{\prime}$ is contained in a simple closed curve in $\Gamma^{\prime}$, which intersects $D_{1}$ transversely in some point in $\operatorname{int}\left(\varepsilon_{1}^{\prime}\right)$. Since $D_{1} \cup D_{2}$ is a sphere and $\Gamma^{\prime} \cap \partial B_{2} \subset \varepsilon_{2}, D_{2}$ intersects $\varepsilon_{2}$ transversely in a single interior point. Thus $D_{1} \cup D_{2}$ is a bridging sphere for $\Gamma^{\prime}$ between $\varepsilon_{1}^{\prime}$ and $\varepsilon_{2}$. Since $D_{1} \cap \varepsilon_{1}=D_{1} \cap \varepsilon_{1}^{\prime}, D_{1} \cup D_{2}$ is also a bridging sphere for $\Gamma$ between $\varepsilon_{1}$ and $\varepsilon_{2}$. But this contradicts our hypothesis. Thus, as an edge of $\Gamma^{\prime}, \varepsilon_{2}$ cannot contain $K_{2}$.

Observe that a 3-connected embedded graph $\Gamma$ can have no bridging spheres. Thus, by the Local Knotting Lemma, adding a local knot $\kappa_{1}$ to any edge of $\Gamma$ does not cause any other edge of $\Gamma$ to contain a new local knot $\kappa_{2}$.

Proposition 4. Let $\Delta$ be an embedded 3-connected graph, and let $H$ be a subgroup of $\mathrm{TSG}_{+}(\Delta)$ which is induced by an isomorphic subgroup $G$ of $\mathrm{Diff}_{+}\left(S^{3}\right)$ and such that no non-trivial element of $H$ fixes any vertex of $\Delta$. Then $\Delta$ can be re-embedded as $\Gamma$ such that $H=\mathrm{TSG}_{+}(\Gamma)$ and $H$ is induced by $G$.

Proof. The vertices of $\Gamma$ will be the same as those of $\Delta$. We will obtain the edges of $\Gamma$ by adding local knots to the edges of $\Delta$. For each $a \in H$, let $g_{a}$ denote the unique element of $G$ which induces $a$ on $\Delta$. Since $G$ is a finite subgroup of $\operatorname{Diff}_{+}\left(S^{3}\right)$, we can choose a neighborhood $N(\Delta)$ so that $N(V)$ and $N(E)$ are each setwise invariant under $G$.

Let $\left\{\delta_{1}, \ldots, \delta_{n}\right\}$ consist of one representative from each edge orbit of $\Delta$ under $H$. Without loss of generality, we can assume there is some $m \leqslant n$ such that $\delta_{i}$ is invertible if and only if $i \leqslant m$. Since no non-trivial element of $H$ fixes any vertex, 
for each $i \leqslant m$, there is a unique $a_{i} \in H$ such that $g_{a_{i}}$ inverts $\delta_{i}$, and there is no non-trivial element of $G$ which leaves any $\delta_{i}$ setwise invariant without inverting it.

Let $\left\{\kappa_{1}, \ldots, \kappa_{n}\right\}$ be a set of distinct prime knots none of which is contained in $\Delta$, such that if $i \leqslant m$ then $\kappa_{i}$ is strongly invertible and otherwise $\kappa_{i}$ is non-invertible. For each $i$, we add the local knot $\kappa_{i}$ to $\delta_{i}$ with ball $N\left(\delta_{i}\right)$ and call this new embedded edge $\varepsilon_{i}$. For $i \leqslant m$, since $\kappa_{i}$ is strongly invertible, we can add $\kappa_{i}$ to $\delta_{i}$ in such a way that $g_{a_{i}}\left(\varepsilon_{i}\right)=\varepsilon_{i}$.

If $a$ and $b$ are elements of $H$ such that $g_{a}\left(\delta_{i}\right)=g_{b}\left(\delta_{i}\right)$, then $g_{a}\left(\varepsilon_{i}\right)=g_{b}\left(\varepsilon_{i}\right)$. For each edge $e$ of $\Delta, e$ is in the orbit of a unique $\delta_{i}$. Now there is an $a \in H$ such that $e=a\left(\delta_{i}\right)$. So we can re-embed $e$ as $g_{a}\left(\varepsilon_{i}\right)$ and this embedding does not depend on our choice of $a$. Since the local knots were each added within $N(E)$, the interiors of the new edges are pairwise disjoint. Let $\Gamma$ denote the embedded graph obtained from $\Delta$ by re-embedding the edges in this way. Observe that the local knot $\kappa_{i}$ was added to an edge $e$ of $\Delta$ if and only if $e$ is in the orbit of $\delta_{i}$ under $G$. By our construction, for every $a \in H, \Gamma$ is setwise invariant under $g_{a}$. Thus $H$ is a subgroup of $\mathrm{TSG}_{+}(\Gamma)$ which is induced by $G$. Now it follows from the Local Knotting Lemma that an embedded edge $\varepsilon$ of $\Gamma$ contains the local knot $\kappa_{i}$ if and only if $\varepsilon$ is in the orbit of $\varepsilon_{i}$ under $G$.

We show as follows that $H=\mathrm{TSG}_{+}(\Gamma)$. Let $\varphi$ be a non-trivial element of $\mathrm{TSG}_{+}(\Gamma)$. Then there is some diffeomorphism $h:\left(S^{3}, \Gamma\right) \rightarrow\left(S^{3}, \Gamma\right)$ such that $h$ induces $\varphi$ on $\Gamma$. Since $\varphi$ is non-trivial, there is some edge $\varepsilon$ which $h$ does not leave setwise invariant. For some $i, \varepsilon$ is in the orbit of $\varepsilon_{i}$ under $G$, and hence contains the local knot $\kappa_{i}$. Thus $h(\varepsilon)$ must also contain the local knot $\kappa_{i}$, and therefore $h(\varepsilon)$ is also in the orbit of $\varepsilon_{i}$ under $G$. Since $\varepsilon$ and $h(\varepsilon)$ are both in the orbit of $\varepsilon_{i}$ under $G$, there is some $g_{1} \in G$ such that $g_{1}(\varepsilon)=h(\varepsilon)$. Thus $g_{1}^{-1} \circ h(\varepsilon)=\varepsilon$.

We define another diffeomorphism $f:\left(S^{3}, \Gamma\right) \rightarrow\left(S^{3}, \Gamma\right)$ as follows. If $g_{1}^{-1} \circ h$ inverts $\varepsilon$, then the local knot $\kappa_{i}$ must be invertible. Thus there is some element of $G$ which inverts $\varepsilon_{i}$, and since $\varepsilon$ is in the orbit of $\varepsilon_{i}$ under $G$ there is some $g_{2} \in G$ which inverts $\varepsilon$. In this case we let $f=g_{2} \circ g_{1}^{-1} \circ h$. Otherwise we let $f=g_{1}^{-1} \circ h$. Thus in either case, $f(\varepsilon)=\varepsilon$, fixing both vertices of $\varepsilon$.

We will show below that $f$ actually fixes every vertex of $\Gamma$. Since we have shown that $f$ fixes the vertices of $\varepsilon$, it suffices to show that if $f$ fixes a vertex $x$, then $f$ fixes every vertex adjacent to $x$. Suppose that there is some edge $\varepsilon^{\prime}$ containing the vertex $x$, such that $f\left(\varepsilon^{\prime}\right) \neq \varepsilon^{\prime}$. By the same argument given two paragraphs up, since $\varepsilon^{\prime}$ and $f\left(\varepsilon^{\prime}\right)$ contain the same local knots, there is some $g_{3} \in G$ such that $g_{3}\left(\varepsilon^{\prime}\right)=f\left(\varepsilon^{\prime}\right)$. By hypothesis no non-trivial element of $G$ fixes any embedded vertex. Let $x^{\prime}$ denote the vertex of $g_{3}\left(\varepsilon^{\prime}\right)$ other than $x$, then $g_{3}(x)=x^{\prime}$. So $g_{3}^{-1} \circ f$ is a diffeomorphism of $\left(S^{3}, \Gamma\right)$ that takes $\varepsilon^{\prime}$ to itself interchanging $x$ and $x^{\prime}$. It follows that the local knot which is contained in $\varepsilon^{\prime}$ must be invertible. Hence, as in the above paragraph, there is a $g_{4} \in G$ which inverts $\varepsilon^{\prime}$. But this implies that $g_{3} \circ g_{4}\left(\varepsilon^{\prime}\right) \neq \varepsilon^{\prime}$ and $g_{3} \circ g_{4}(x)=x$. So $g_{3} \circ g_{4}$ is a non-trivial element of $G$ that fixes the vertex $x$ of $\Delta$. This contradicts 
the hypothesis of the proposition. Hence $f$ fixes every vertex of $\Gamma$.

Recall that either $f=g_{2} \circ g_{1}^{-1} \circ h$ or $f=g_{1}^{-1} \circ h$. Since $f$ fixes every vertex of $\Gamma, h$ induces the same automorphism on $\Gamma$ as either $g_{1} \circ g_{2}^{-1}$ or $g_{1}$ does. Since the automorphisms of $\Gamma$ induced by both $g_{1} \circ g_{2}^{-1}$ and $g_{1}$ are elements of $H$, it follows that $\varphi$, the automorphism of $\Gamma$ induced by $h$, is also an element of $H$. Therefore $\mathrm{TSG}_{+}(\Gamma)=H$.

The following result follows immediately from Propositions 1 and 4, together with the fact that a 3-connected embedded graph has no type I, II, or III spheres.

Corollary. Let $\Delta$ be an embedded 3-connected graph. Let $H \leq \mathrm{TSG}_{+}(\Delta)$ such that no non-trivial element of $H$ fixes any vertex of $\Delta$. Then $\Delta$ can be re-embedded as $\Gamma$ such that $H=\mathrm{TSG}_{+}(\Gamma)$ and $H$ is induced by an isomorphic finite subgroup of $\operatorname{Diff}_{+}\left(S^{3}\right)$.

We shall use Proposition 4 and the Graph Embedding Lemma to prove the following converse of Theorem 2. Note that the statement of Theorem 3 that we prove below is slightly stronger than that given in the introduction.

Theorem 3. For every finite subgroup $G$ of $\operatorname{Diff}_{+}\left(S^{3}\right)$, there is an embedded 3connected graph $\Gamma$ such that $G \cong \mathrm{TSG}_{+}(\Gamma)$ and $\mathrm{TSG}_{+}(\Gamma)$ is induced by G. Moreover, $\Gamma$ can be chosen to be a complete bipartite graph $K_{n, n}$ for some $n$.

Proof. The complete bipartite graph $K_{3,3}$ is 3 -connected. By the corollary there is an embedded graph $\Gamma$ with underlying abstract graph $K_{3,3}$ such that $\mathrm{TSG}_{+}(\Gamma)$ is trivial. So we assume that the group $G$ is not trivial.

Now let $n$ denote the order of $G$. First we suppose that $n>2$. Let $\left\{v_{1}, v_{2}, \ldots, v_{n}\right\}$ and $\left\{w_{1}, w_{2}, \ldots, w_{n}\right\}$ denote the sets of vertices of an abstract complete bipartite graph $K_{n, n}$. Since $n>2$ we know that $K_{n, n}$ is 3 -connected. Pick $x_{1}$ to be a point in $S^{3}$ that is not fixed by any non-trivial element of $G$. Let $\left\{x_{1}, x_{2}, \ldots, x_{n}\right\}$ denote the orbit of $x_{1}$ under $G$. We will define a homomorphism $\Psi: G \rightarrow \operatorname{Aut}\left(K_{n, n}\right)$ as follows. Let $g \in G$. Then for every $i \leq n$, there is a $j \leq n$ such that $g\left(x_{i}\right)=x_{j}$. We define $\Psi(g)\left(v_{i}\right)=v_{j}$ and $\Psi(g)\left(w_{i}\right)=w_{j}$. Let $H$ denote the image of $\Psi$.

Since no non-trivial element of $G$ fixes any $x_{i}, \Psi$ is one-to-one, and hence $H \cong G$. Also, no non-trivial element of $H$ takes any vertex to itself. Furthermore, since no element of $H$ takes any $v_{i}$ to any $w_{j}$, no edges of $K_{n, n}$ are inverted by any element of $H$. Now we can apply the Graph Embedding Lemma to obtain an embedded graph $\Gamma$ with underlying abstract graph $K_{n, n}$ such that $G$ induces $H$ on $\Gamma$. Furthermore, by Proposition 4, $\Gamma$ can be chosen so that $H=\mathrm{TSG}_{+}(\Gamma)$ and $H$ is induced by $G$.

Finally, suppose that $n=2$, so $G \cong \mathbb{Z}_{2}$. Let $\left\{v_{1}, v_{2}, v_{3}, v_{4}\right\}$ and $\left\{w_{1}, w_{2}, w_{3}, w_{4}\right\}$ denote the sets of vertices of an abstract complete bipartite graph $K_{4,4}$. Then $K_{4,4}$ is 3-connected. Let $H$ be the subgroup of $\operatorname{Aut}\left(K_{4,4}\right)$ generated by the element 
$\left(v_{1}, v_{2}\right)\left(v_{3}, v_{4}\right)\left(w_{1}, w_{2}\right)\left(w_{3}, w_{4}\right)$. Then $H \cong \mathbb{Z}_{2}$, no vertex of $K_{4,4}$ is fixed by any non-trivial element of $H$, and there are no edges which are inverted by an element of $H$. Thus we can again apply the Graph Embedding Lemma and Proposition 4 to get an embedded graph $\Gamma$ with underlying abstract graph $K_{4,4}$ such that $\mathrm{TSG}_{+}(\Gamma)$ is induced by $G$ and $G \cong \mathrm{TSG}_{+}(\Gamma)$.

\section{References}

[B1] L. Babai, Automorphism groups of planar graphs, I. Discrete Math. 2 (1972), 295-307. Zbl 0238.05111 MR 0302494

[B2] L. Babai, Automorphism groups of planar graphs, II. In Infinite and finite sets (Colloq., Keszthely, 1973; dedicated to P. Erdös on his 60th birthday) Vol I, Colloq. Math. Soc. Janos Bolyai 10, North-Holland, Amsterdam 1975, 29-84.Zbl 0301.05116 MR 0371715

[B3] L. Babai, On the abstract group of automorphisms. In Combinatorics (Swansea 1981), London Math. Soc. Lecture Note Ser. 52, Cambridge University Press, Cambridge, New York 1981, 1-40. Zbl 0467.05031 MR 0633647

[B4] L. Babai, Automorphism groups, isomorphism, reconstruction. In Handbook of Combinatorics 2, Elsevier, Amsterdam 1995, 1447-1540. Zbl 0846.05042 MR 1373683

[D] R. Diestel, Graph Theory. Grad. Texts in Math. 173, Second edition, Springer-Verlag, New York 2000. Zbl 0945.05002 MR 1743598

[DV] P. Du Val, Homographies, Quaternions and Rotations. Oxford Mathematical Monographs, Clarendon Press, Oxford 1964. Zbl 0128.15403 MR 0169108

[F1] E. Flapan, Symmetries of Möbius ladders. Math. Ann. 283 (1989), 271-283. Zbl 0642.57008 MR 0980598

[F2] E. Flapan, Rigidity of graph symmetries in the 3-sphere. J. Knot Theory Ramifications 4 (1995), 373-388. Zbl 0837.57002 MR 1347360

[Fr] R. Frucht, Herstellung von Graphen mit vorgegebener abstrakter Gruppe. Compositio Math. 6 (1938), 239-250. Zbl 0020.07804

[Ha] R. Hartley, Knots with free period. Canadian J. Math. 33 (1981), 91-102.Zbl 0481.57003 MR 0608857

[JS] W. Jaco and P. Shalen, Seifert fibred spaces in 3-manifolds. Mem. Amer. Math. Soc. 220, Amer. Math. Soc., Providence, RI, 1979. Zbl 0415.57005 MR 0539411

[Jo] K. Johannson, Homotopy equivalences of 3-manifolds with boundaries. Lecture Notes in Math. 761, Springer-Verlag, New York, Berlin, Heidelberg 1979. Zbl 0412.57007 MR 0551744

[L] H. C. Longuet-Higgins, The symmetry groups of non-rigid molecules. Molec. Phys. 6 (1963), 445-460. Zbl 0121.45702

[M] P. Mani, Automorphismen von polyedrischen Graphen. Math. Ann. 192 (1971), 279-303. Zbl 0206.51402 MR 0296808

[Ma] W. K. Mason, Homeomorphic continuous curves in 2-space are isotopic in 3-space. Trans. Amer. Math. Soc. 142 (1969), 269-290. Zbl 0183.51403 MR 0246276 
[Mi] J. Milnor, Groups which act on $S^{n}$ without fixed points. Amer. J. Math. 79 (1957), 623-630. Zbl 0078.16304 MR 0090056

[Moi] E. E. Moise, Geometric Topology in Dimensions 2 and 3. Grad. Texts in Math. 47, Springer-Verlag, New York 1977. Zbl 0349.57001 MR 0488059

[MB] J. Morgan and H. Bass, The Smith Conjecture. Pure Appl. Math. 112, Academic Press, Orlando 1984. Zbl 0599.57001 MR 0758460

[Mo] G. Mostow, Strong Rigidity for Locally Symmetric Spaces. Ann. of Math. Stud. 78, Princeton University Press, Princeton 1973. Zbl 0265.53039 MR 0385004

[Sa] G. Sabidussi, Graphs with given group and given graph-theoretical properties. Canad. J. Math. 9 (1957), 515-525. Zbl 0079.39202 MR 0094810

[Si] J. Simon, A topological approach to the stereochemistry of nonrigid molecules. In Graph theory and topology in chemistry (Athens, Ga. 1987), Stud. Phys. Theoret. Chem. 51, Elsevier, Amsterdam 1987, 43-75. Zbl 0668.57006 MR 0941547

[Sm] P. A. Smith, Transformations of finite period II. Ann. of Math. 40 (1939), 690-711. Zbl 0021.43002 MR 0000177

[Su] S. Suzuki, A prime decomposition theorem for a graph in the 3-sphere. In Topology and Computer Science (Atami 1986), Kinokuniya, Tokyo 1987, 259-276. MR1112597

[Th] W. Thurston, Three-dimensional manifolds, Kleinian groups and hyperbolic geometry. Bull. Amer. Math. Soc. 6 (1982), 357-381. Zbl 0496.57005 MR 0648524

[Tr] H. F. Trotter, Periodic automorphisms of groups and knots. Duke Math J. 28 (1961), 553-557. Zbl 0109.41604 MR 0133820

[Wa] F. Waldhausen, On irreducible 3-manifolds which are sufficiently large. Ann. Math. 87 (1968), 56-88. Zbl 0157.30603 MR 0224099

[Z] B. Zimmerman, On finite simple groups acting on homology 3-spheres. Topology Appl. 125 (2002), 199-202. Zbl 1012.57028 MR 1933571

Received July 9, 2003

Erica Flapan, Department of Mathematics, Pomona College, Claremont, CA 91711, U.S.A.

E-mail: eflapan@pomona.edu

Ramin Naimi, Department of Mathematics, Occidental College, Los Angeles, CA 90041, U.S.A.

E-mail: rnaimi@oxy.edu

James Pommersheim, Department of Mathematics, Reed College, Portland, OR 97202, U.S.A.

E-mail: jamie@reed.edu

Harry Tamvakis, Department of Mathematics, Brandeis University - MS 050, P.O. Box 9110, Waltham, MA 02454-9110, U.S.A.

E-mail: harryt@brandeis.edu 